\title{
Possible interpretation of the precession of comet 67P/Churyumov-Gerasimenko
}

\author{
P. J. Gutiérrez ${ }^{1}$, L. Jorda ${ }^{2}$, R. W. Gaskell ${ }^{3}$, B. J. R. Davidsson ${ }^{4}$, C. Capanna ${ }^{2}$, S. F. Hviid ${ }^{5}$, H. U. Keller ${ }^{6}$, L. Maquet ${ }^{7}$, \\ S. Mottola ${ }^{5}$, F. Preusker ${ }^{5}$, F. Scholten ${ }^{5}$, L. M. Lara ${ }^{1}$, F. Moreno ${ }^{1}$, R. Rodrigo ${ }^{8,9}$, H. Sierks ${ }^{10}$, C. Barbieri ${ }^{11}$, P. Lamy ${ }^{2}$, \\ D. Koschny ${ }^{12}$, H. Rickman ${ }^{4,13}$, J. Agarwal ${ }^{10}$, M. F. A'Hearn ${ }^{14}$, A. T. Auger ${ }^{2}$, M. A. Barucci ${ }^{15}$, J. L. Bertaux ${ }^{16}$, \\ I. Bertini ${ }^{17}$, G. Cremonese ${ }^{18}$, V. Da Deppo ${ }^{19}$, S. Debei ${ }^{20}$ M. De Cecco $^{21}$, M. R. El-Maarry ${ }^{22}$, S. Fornasier ${ }^{15}$, M. Fulle ${ }^{23}$, \\ O. Groussin ${ }^{2}$, P. Gutiérrez-Marques ${ }^{10}$, C. Güttler ${ }^{10}$, W. H. Ip ${ }^{24}$ J. Knollenberg ${ }^{5}$ J. R. Kramm ${ }^{10}$, E. Kührt ${ }^{5}$, \\ M. Küppers ${ }^{25}$, F. La Forgia ${ }^{11}$ M. Lazzarin ${ }^{11}$ J. J. López-Moreno ${ }^{1}$, S. Magrin ${ }^{11}$, S. Marchi ${ }^{26}$, F. Marzari ${ }^{11}$, \\ G. Naletto ${ }^{20,17,19}$, N. Oklay ${ }^{10}$, M. Pajola ${ }^{17}$, A. Pommerol ${ }^{22}$, D. Sabau ${ }^{27}$, N. Thomas ${ }^{22,28}$, I. Toth ${ }^{29}$, \\ C. Tubiana ${ }^{10}$, and J. B. Vincent ${ }^{10}$ \\ (Affiliations can be found after the references)
}

Received 22 December 2015 / Accepted 15 March 2016

\begin{abstract}
Context. Data derived from the reconstruction of the nucleus shape of comet 67P/Churyumov-Gerasimenko (67P) from images of the OSIRIS camera onboard ROSETTA show evidence that the nucleus rotates in complex mode. First, the orientation of the spin axis is not fixed in an inertial reference frame, which suggests a precessing motion around the angular momentum vector with a periodicity of approximately $257 \mathrm{~h} \pm 12 \mathrm{~h}$. Second, periodograms of the right ascension and declination (RA/Dec) coordinates of the body-frame $Z$ axis show a very significant (higher than $99.99 \%$ ) periodicity at $276 \mathrm{~h} \pm 12 \mathrm{~h}$, different from the rotational period of $12.40 \mathrm{~h}$ as previously determined from light-curve analysis.

Aims. The main goal is to interpret the data and associated periodicities of the spin axis orientation in space.

Methods. We analyzed the spin axis orientation in space and associated periodicities and compared them with solutions of Euler equations under the assumption that the body rotates in torque-free conditions. Statistical tests comparing the observationally derived spin axis orientation with the outcome from simulations were applied to determine the most likely inertia moments, excitation level, and periods.

Results. Under the assumption that the body is solid-rigid and rotates in torque-free conditions, the most likely interpretation is that 67P is spinning around the principal axis with the highest inertia moment with a period of about $13 \mathrm{~h}$. At the same time, the comet precesses around the angular momentum vector with a period of about $6.35 \mathrm{~h}$. While the rotating period of such a body would be about $12.4 \mathrm{~h}$, RA/Dec coordinates of the spin axis would have a periodicity of about $270 \mathrm{~h}$ as a result of the combination of the two aforementioned motions.

Conclusions. The most direct and simple interpretation of the complex rotation of $67 \mathrm{P}$ requires a ratio of inertia moments significantly higher than that of a homogeneous body.
\end{abstract}

Key words. comets: general - comets: individual: 67P/Churyumov-Gerasimenko

\section{Introduction}

Immediately after the wake-up of ROSETTA and the recommissioning of the scientific instruments, the OSIRIS cameras (Keller et al. 2007) started to perform systematic observations of comet 67P/Churyumov-Gerasimenko (67P). One of the goals of these observations was to accurately determine the rotational parameters of $67 \mathrm{P}$ and establish a solid starting point for detecting possible variations. By using data from March to June, 2014, Mottola et al. (2014) analyzed these early photometric observations and obtained that $67 \mathrm{P}$ was rotating at this epoch with a period of $12.4043 \pm 0.0007 \mathrm{~h}$. Near-nucleus operations began in early August 2014, with a comet-characterization phase followed by a mapping phase. One of the main goals during those two phases was to reconstruct detailed topographic models of the nucleus of 67P and to retrieve its global shape by using the hundreds of images that the OSIRIS instrument acquired.

So far, two different methods have been applied to retrieve high-resolution shape models of 67P from OSIRIS images: stereo-photogrammetry (SPG; Preusker et al. 2015) and stereophotoclinometry (SPC; Jorda et al. 2016). The reconstruction of the shape of $67 \mathrm{P}$ involves a very accurate determination using stereo landmarks of the comet position and attitude in camera frame. Combined with the knowledge of the S/C position and attitude reconstructed by the Rosetta Mission Operations Centre (RMOC) from the radio ranging and star tracker data, this provides a very accurate determination of the comet position and attitude in an inertial frame, usually the EME2000 reference frame. Preusker et al. (2015) described the reconstruction of the so-called SHAP4S model using the SPG technique. They showed that the accuracy of the retrieved topographic model significantly improves when a precessing spin axis is introduced. By defining seven different image blocks, Preusker et al. (2015) obtained that the right ascension and declination (RA/Dec) of the spin pole that allow the most accurate shape model describe a circle around $(\mathrm{RA}, \mathrm{Dec})=\left(69.54^{\circ} \pm 0.1^{\circ}, 64.11^{\circ} \pm 0.05^{\circ}\right)$, with a radius of $0.14^{\circ} \pm 0.03^{\circ}$ and a possible period of $256.8 \mathrm{~h} \pm 12 \mathrm{~h}$ (10.7 d \pm 0.5 d), see Fig. 2 in Preusker et al. (2015). This was interpreted by the authors as the precession of the spin axis around the angular momentum vector. It is the first evidence that comet $67 \mathrm{P}$ might be rotating in complex mode, although with a low excitation given the small angle between the spin axis and the presumable angular momentum vector.

The results obtained by Jorda et al. (2016) with the SPC method are consistent with those of Preusker et al. (2015). 


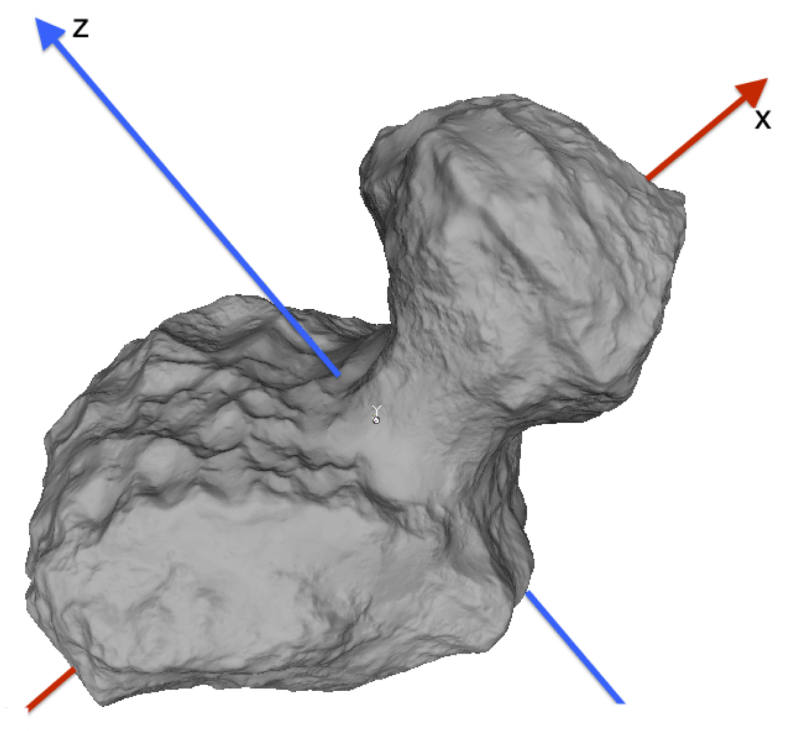

Fig. 1. SPC shape of $67 \mathrm{P} /$ Churyumov-Gerasimenko (SHAP5, version 1.2, Jorda et al. 2016) in the so-called Cheops frame (see Scholten et al. 2015). The spin axis is the $z$-axis.

By applying the SPC method, Jorda et al. (2016) retrieved a spin axis that moves around $(\mathrm{RA}, \mathrm{Dec})=\left(69.57^{\circ},+64.01^{\circ}\right)$. Jorda et al. (2016) obtained that the spin axis does not describe a circumference, but approximately fills an ellipse in an isotropic plot (Fig. 2). This difference might arise because the data from SPC method cover a time span much longer than those of the SPG method. When SPC and SPG results are compared, the putative coordinates of the angular momentum vector are also slightly different by about $0.1^{\circ}$. This small difference, slightly larger than the associated errors, still needs to be understood (Jorda et al. 2016). Interestingly, Jorda et al. (2016) analyzed the spin axis orientation (the body frame $z$-axis coordinates) by means of the phase-dispersion minimization technique (Stellingwerf 1978) and obtained a confident periodicity of $276 \mathrm{~h} \pm 12 \mathrm{~h}$ $(11.5 \mathrm{~d} \pm 0.5 \mathrm{~d})$ from separately considering the RA and Dec coordinates. This periodicity can be considered to agree within the error bars with the early determination by Preusker et al. (2015). Additionally, among other characteristics of 67P, Jorda et al. (2016) determined the pre-perihelion rotational period and its evolution with time, obtaining that up to December 2014 it was $12.4041 \mathrm{~h} \pm 0.0001 \mathrm{~h}$, which refines the earlier determination of Mottola et al. (2014) that was based on light curves. This means that the SPG and SPC results both point to a situation in which the motion of the spin pole is perturbed by an additional periodic motion with a period of $\sim 270 \mathrm{~h}$, much longer than the spin period of $12.40 \mathrm{~h}$ measured by Mottola et al. (2014). This seems to confirm the complex nature of the rotation of 67P, but an interpretation of these measurements is still lacking.

The goal of this paper is to interpret the periodicity associated with the spin axis orientation measured by ROSETTA taking into account the pre-perihelion rotation period measured by Mottola et al. (2014) that was later refined by Jorda et al. (2016). We restrict our analysis to the observations obtained until the end of December 2014 (250 days before perihelion), during which the rotation period remained constant (within the accuracy of the OSIRIS and flight dynamics measurements). After this, the rotation period started to vary (Jorda et al. 2016; Rosetta

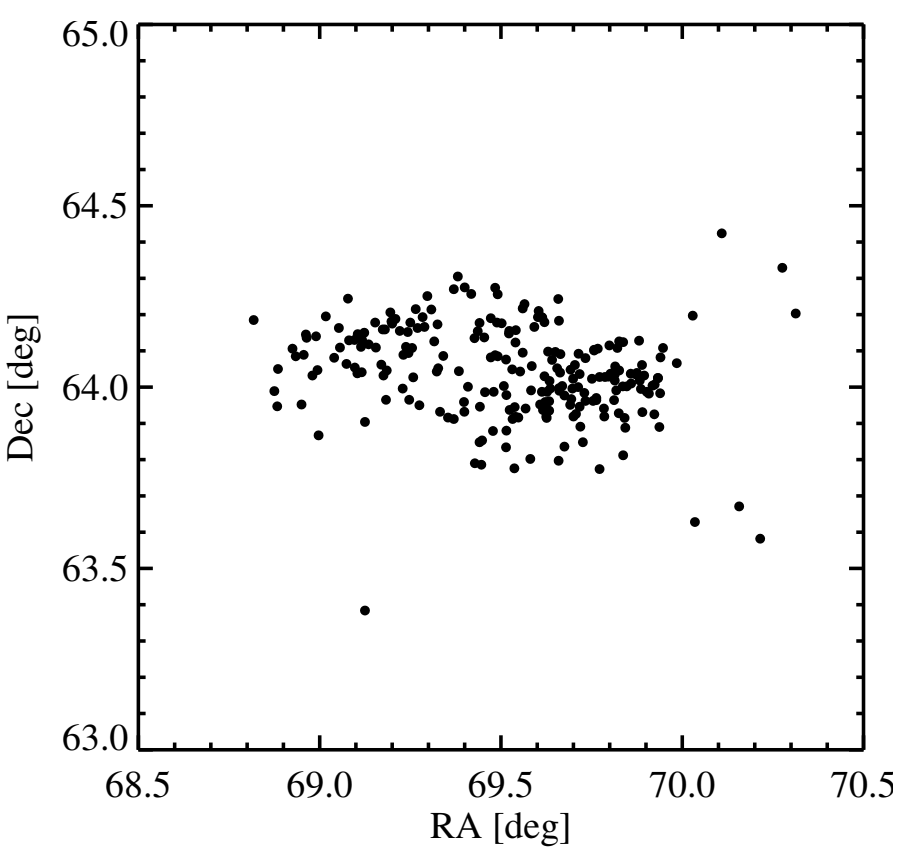

Fig. 2. Coordinates in $\mathrm{J} 2000$ of the spin axis of $67 \mathrm{P}$ for the time interval between -376 and -250 days from perihelion. These coordinates are obtained during the SPC procedure employed to retrieve the shape of 67P (Jorda et al. 2016).

Mission Operations Reports), indicating that an outgassinginduced torque started to have detectable effects on the angular momentum of comet 67P. An activity-driven torque has been simulated (see, e.g., Bertaux 2015; Keller et al. 2015) to explain the spin period evolution of 67P. These simulations and calculations suggest that the sublimation-induced torque is negligible at large heliocentric distances and does not affect the rotational evolution. We therefore assume in the following that $67 \mathrm{P}$ was rotating under torque-free conditions before December 2014.

Finally, the moments of inertia can be derived from the geometric global shape of 67P assuming it has a homogeneous density. Considering the latest SHAP5 model (SPC method, Jorda et al. 2016), the principal inertia moments would follow the relationship $I_{x}: I_{y}: I_{z}=1.00: 1.83: 1.98$, where $I_{x}, I_{y}$, and $I_{z}$ are the inertia moments along the largest, intermediate, and shortest principal axes ${ }^{1}$, respectively.

\section{Data}

Two very different data sets of the spin axis orientation are available: those obtained with the SPG method on the one hand, and those obtained with the SPC on the other hand. The first data set includes seven determinations considering time intervals (blocks) of 12-13 h and covering a time span of about one month. The SPC method yielded 232 estimates of the spin axis orientation that cover a time span of approximately 125 days (from August 2014 to December 2014), each of them defined for time intervals of $10 \mathrm{~h}$. This means that the coordinates do not correspond to the instantaneous position, but rather to its average location within a time interval of $10 \mathrm{~h}$.

\footnotetext{
1 At the time the simulations of this study were performed, the inertia moments derived assuming homogeneity were those reported above. Later on, Jorda et al. (2016) refined the estimation of the moments of inertia and obtained 1:1.85:1.99. These small differences do not affect the present study.
} 
The image blocks (set of images allowing the retrieval of the average spin axis orientation for the covered time interval) in the two data sets are slightly different, therefore we considered only the data set derived from SPC to simplify the procedures. This was done with the understanding that data from SPG are compatible with those of SPC and that the addition of the seven data points from SPG to the 232 spin axis orientations from SPC will not change the conclusions of the study. The RA/Dec coordinates of the body frame $z$-axis as retrieved by means of the SPC method considered here are shown in Fig. 2. The typical formal angular error associated with RA and Dec coordinates is on the order of $0.03^{\circ}$ (Jorda et al. 2016). Because our intention is to perform statistical tests by comparing observational and theoretical distributions, we decided to remove seven clearly identifiable outliers from these coordinates and kept 225 coordinates. This data set is called observationally derived data.

In Fig. 2 we show that the spin axis orientation is not constant, but varies in time around a central position. Relying on the accuracy of the SPC method, we consider that this might be a clear indication that the body is slightly excited from the rotational point of view, with the spin axis moving around the constant angular momentum orientation. When estimated as the mean value of the spin axis coordinates, the RA and Dec of the angular momentum vector would be around $69.5^{\circ}$ and $64.04^{\circ}$, respectively. These coordinates are slightly different from those reported by Jorda et al. (2016) (and slightly closer to those of Preusker et al. 2015). The small difference arises because outliers were removed from our data set.

If RA and Dec coordinates, considered separately, are analyzed by means of different time-series techniques, a very significant peak at periodicities around $270 \mathrm{~h}$ appears. Figure 3 shows the Lomb ${ }^{2}$ periodograms (Lomb 1976) of the RA and Dec data displayed in Fig. 2. The Lomb periodogram shows the highest significance of RA data for a periodicity of approximately $264 \mathrm{~h}$, while Dec data have a significant associated periodicity at approximately $270 \mathrm{~h}$, both compatible with the periodicity of $276 \mathrm{~h}$ obtained by Jorda et al. (2016) with the PDM technique. In addition to this periodicity, it is known that the light curve has a periodicity of $12.40 \mathrm{~h}$ (Mottola et al. 2014), which was interpreted as the spin period, assuming that $67 \mathrm{P}$ was rotating in pure spin mode at that time. In principle, the $12.40 \mathrm{~h}$ might well correspond to the modulus of full rotational vector, $\boldsymbol{\Omega}$, the rotational period. Determinations of the omega vector modulus obtained with the SPC method (Jorda et al. 2016) point out that this interpretation is probably correct. Nevertheless, this still needs some clarification and is discussed below. In addition, the meaning of the $270 \mathrm{~h}$ periodicity found in the spin axis RA/Dec coordinates needs to be determined, as well as the information that might be extracted from it. The $270 \mathrm{~h}$ periodicity is called RA/Dec periodicity throughout.

\section{Some considerations from Euler equations}

Misunderstandings may arise when the rotation of a body in space is described with different references frames. The reference frame of the motion needs to be specified and the transformation between body frame and an inertial reference frame has to be defined. We here use the following terminology. When the motion of the body is described as the rotation around the axes of the body frame, the term "intrinsic" must be used to define

\footnotetext{
2 or Lomb-Scargle. As the implementation used is that of ENVI/IDL which is based on Numerical Recipes (Press et al. 1992) we will use the same notation, calling the method simply Lomb.
}

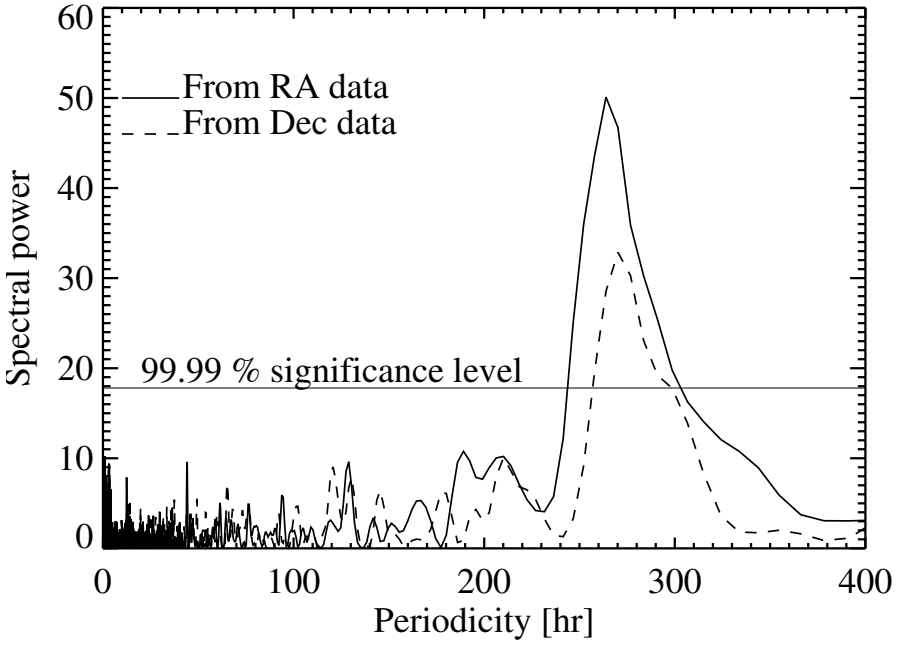

Fig. 3. Lomb periodograms of the RA and Dec coordinates of the spin axis displayed in Fig. 2. A significant peak (with a significance higher than $99.99 \%$ ) is located at a periodicity of approximately $270 \mathrm{~h}$. This result is fully compatible with those found by Jorda et al. (2016) with the PDM technique. Data phased to this periodicity are shown in Fig. 12 of Jorda et al. (2016).

the rotational motion. When the rotation is described as the motion of the inertial frame around a fixed body frame, the rotations are considered as "extrinsic". We define a body frame with the orthogonal axes along the principal axes of inertia. The $x$-axis has the direction of the principal axis associated to the lowest moment of inertia $\left(I_{x}\right)$. The $y$-axis has the direction of the principal axis of inertia associated to the intermediate inertia moment $\left(I_{y}\right)$. Finally, the $z$-axis is the orthogonal axis parallel to the principal axis associated with the hightest inertia moment $\left(I_{z}\right)$. To describe the rotation of this $x y z$ body frame (and therefore that of the body, which is considered to be rigid) in space, a convenient set of Euler angles must be selected. The Euler angles define the transformation matrix between the body frame ( $x y z)$ and the inertial frame $(X Y Z)$. By considering the standard $Z X Z$ convention, the transformation between the body frame $x y z$ and the inertial frame $X Y Z$, and therefore the motion of the body in space, is defined by the standard Euler angles $\phi, \theta$, and $\psi$. These three angles and the corresponding associated velocities define a rotation of the body frame with regard to the inertial frame and have specific meanings. In the terminology we use, the $\psi$ angle represents the intrinsic rotation of the body, which is a spin around the principal axis $z$ that is associated with the highest inertia moment. The $\theta$ angle describes a rotation around the line of nodes defined by the two aforementioned reference frames. This motion is called nutation and can be described as an oscillation of the body frame $z$-axis with regard to the inertial $Z$-axis. Finally, $\phi$, the angle between the line the nodes and the inertial $X$-axis, defines a rotation around the inertial $Z$-axis. If the angular momentum orientation is defined along the inertial $Z$-axis, this latter rotational motion is generally described as the precession of the body frame $z$-axis (i.e., the axis defining the intrinsic rotation of the body) around the angular momentum orientation. These reference frames, angles, and their corresponding velocities, are shown in Fig. 4. We here describe the rotational motion of the body as follows:

- $\phi$ and $\dot{\phi}$ describe the precession of the axis with the highest inertia moment $(z)$ around the angular momentum direction $(Z)$. 


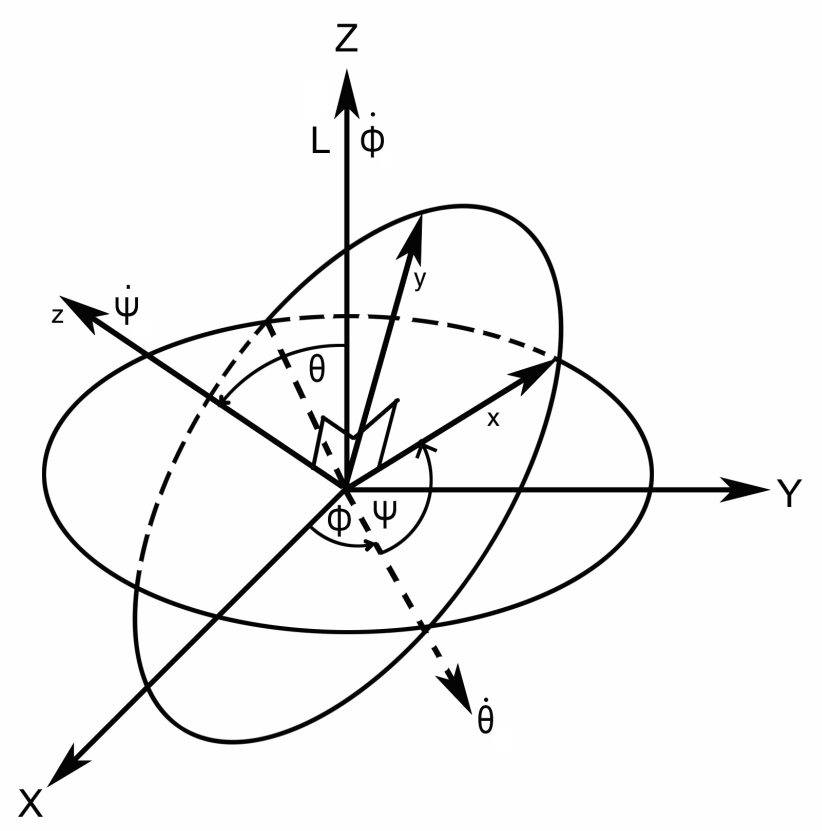

Fig. 4. Body frame $(x y z)$ and inertial frame $(X Y Z)$ with the Euler angles and their velocities in the standard $Z X Z$ convention. This figure is adapted from Fig. A.1 from Samarasinha \& Mueller (2015). Reproduced with permission.

- $\theta$ and $\dot{\theta}$ describe the nutation of the axis with the highest inertia moment $(z)$ with regard to the angular momentum direction $(Z)$.

- $\psi$ and $\dot{\psi}$ represent the intrinsic rotation, spinning, of the body around the axis with the highest inertia moment $(z)$.

- $\mathbf{\Omega}$ is the total rotation of the body, formed by the three previous motions together.

We therefore reserve the use of the term spin to refer to the intrinsic rotation described by the angle $\psi$, and spin axis or spin pole for the body frame $z$-axis. According to this, Fig. 2 shows the RA/Dec coordinates of the spin pole. The term rotation is generally used to describe the combination of all three described motions. Therefore, rotation axis refers to the instantaneous rotation vector driven by the spin, nutation, and precession motions together. The rotation is represented as $\boldsymbol{\Omega}_{X Y Z}$ or $\boldsymbol{\Omega}_{x y z}$, depending on the reference frame in which is expressed. We note that because $\Omega=\left|\boldsymbol{\Omega}_{X Y Z}\right|=\left|\boldsymbol{\Omega}_{x y z}\right|$, the rotational period $P=2 \pi / \Omega$ does not need the reference frame to be specified.

To continue with notations, it is true throughout this study that $I_{x}<I_{y}<I_{z}$. If we call $E$ the rotational energy, $L$ the modulus of the angular momentum vector, the quantity $C=L^{2} / 2 E$ fulfills $I_{x}<C<I_{z}$ in torque-free. We therefore may call the quantity $E_{l}=1-C / I_{z}$ the excitation level $\left(E_{l}\right)$, whose magnitude is 0 when the body rotates at the lowest energy for its angular momentum (i.e., a simple rotation around $z$-axis). When $I_{x}<C<I_{y}$, the body rotates in long-axis mode (LAM). When $I_{y}<C<I_{z}$ is fulfilled, the body rotates in short-axis mode (SAM; see, e.g., Belton 1990). 67P is presumably only slightly excited (given the small amplitude of the very likely complex motion), the rotational excitation must therefore be comparatively low $\left(E_{l} \rightarrow 0, C \rightarrow I_{z}\right)$ and the nucleus probably rotates in SAM, close to its lowest rotational energy for its angular momentum. In SAM, the body spins or intrinsically rotates around the principal axis with the highest inertia moment and at the same time performs both a precession of this principal axis around the angular momentum vector and a nutation, oscillation, also of this principal axis. This means that the previously defined Euler angles are perfectly suitable to describe the possible rotational motion of 67P in SAM.

Under the assumption of torque-free motion, the body must rotate following certain constraints derived from the Euler equations, that is, the Euler angles and their velocities follow some regular behavior depending on inertia moments and excitation level. More details on the description of excited rotational states are given by Samarasinha \& Mueller (2015). The authors included a complete and comprehensive compendium of the equations and their dependences governing the complex motion of a torque-free body in SAM mode. In the following, we summarize the more relevant equations and conclusions for the present study.

Thus, in torque-free and in SAM,

- $\psi$ evolves in a periodic way with a period $P_{\psi}$

$$
P_{\psi}=4 \sqrt{\frac{I_{x} I_{y} I_{z}}{2 E\left(I_{z}-I_{y}\right)\left(\frac{L^{2}}{2 E}-I_{x}\right)}} \int_{0}^{\pi / 2} \frac{\mathrm{d} u}{\sqrt{1-k^{2} \sin ^{2}(u)}},
$$

where

$$
k=\sqrt{\frac{\left(I_{y}-I_{x}\right)\left(I_{z}-\frac{L^{2}}{2 E}\right)}{\left(I_{z}-I_{y}\right)\left(\frac{L^{2}}{2 E}-I_{x}\right)}} .
$$

Even though this is periodic, it does not mean that $\dot{\psi}$ is constant.

- $\dot{\phi}$ is given by

$$
\dot{\phi}=L\left[\frac{\left(I_{z}-I_{y}\right)+\left(I_{y}-I_{x}\right) \operatorname{sn}^{2} \tau}{I_{x}\left(I_{z}-I_{y}\right)+I_{z}\left(I_{y}-I_{x}\right) \operatorname{sn}^{2} \tau}\right],
$$

which is periodic with a period $P_{\psi} / 2$. In the previous expression, $\operatorname{sn} \tau$ is the Jacobian elliptic function of the argument $\tau$ defined as

$$
\tau=t \sqrt{\frac{2 E\left(I_{z}-I_{y}\right)\left(\frac{L^{2}}{2 E}-I_{x}\right)}{I_{x} I_{y} I_{z}}},
$$

and $t$ denotes time.

- $\phi$ is generally aperiodic, except for some particular cases. Nevertheless, a time-averaged period for $\phi$ can be defined as

$$
P_{\phi}=\frac{2 \pi}{\dot{\phi}_{\text {mean }}} \text {. }
$$

- For slightly excited cases, the two above periods fulfill the condition

$$
P_{\psi}>2 P_{\phi}
$$

- $\phi$ and $\psi$ circulate in opposite senses. The component of the total rotational velocity vector, $\boldsymbol{\Omega}_{x y z}$, along the principal axis of inertia with the highest inertia moment is given by $\Omega_{z}=\dot{\phi} \cos (\theta)+\dot{\psi}$.

- $\theta$ oscillates with a period $P_{\psi} / 2$ between a minimum

$$
\theta_{\min }=\cos ^{-1}\left(\sqrt{\frac{I_{z}\left(\frac{L^{2}}{2 E}-I_{x}\right)}{\frac{L^{2}}{2 E}\left(I_{z}-I_{x}\right)}}\right)
$$


and a maximum

$$
\theta_{\max }=\cos ^{-1}\left(\sqrt{\frac{I_{z}\left(\frac{L^{2}}{2 E}-I_{y}\right)}{\frac{L^{2}}{2 E}\left(I_{z}-I_{y}\right)}}\right) .
$$

As two periodicities have been detected for $67 \mathrm{P}$, the $12.40 \mathrm{~h}$ from Mottola et al. (2014) and the RA/Dec periodicity of about $270 \mathrm{~h}$, the first and most intuitive interpretation is to consider that the body spins with a period of $P_{\psi}=12.40 \mathrm{~h}$ while the body frame $z$-axis precesses around the angular momentum vector with a precession period $P_{\phi} \approx 270 \mathrm{~h}$. Nevertheless, in the standard terminology, this description is not supported by the Euler equations, in which, considering the aforementioned constraints, $P_{\psi}$, the spin period, must always be longer than the precession period, $P_{\phi}$, particularly $P_{\psi}>2 P_{\phi}$. Therefore, the RA/Dec periodicity can only be identified with $P_{\psi}$, that is, with the intrinsic rotation period or spin, the rotation of the body around the principal axis with the highest inertia moment. Several combinations of these motions may result in the two observationally detected periodicities. The first problem is to correctly identify the meaning of the $12.40 \mathrm{~h}$ periodicity detected by Mottola et al. (2014) that was confirmed by Jorda et al. (2016). If we assume that $12.40 \mathrm{~h}$ is the rotational period, $P=2 \pi / \Omega$, and that the excitation is comparatively low, we can verify that the precession period, $P_{\phi}$, must be shorter than P. Thus, if $P=12.40 \mathrm{~h}, P_{\phi}<12.40 \mathrm{~h}$, and the $270 \mathrm{~h}$ periodicity can be identified with the spin period, $P_{\psi}$, or any other possible combination of periodicities. We call this case 1 , and it is characterized by $P=12.40 \mathrm{~h}$ and $P_{\phi}<12.40 \mathrm{~h}$ An alternative to this possibility arises if the periodicity detected by Mottola et al. (2014) that was confirmed by Jorda et al. (2016) is not the rotation period. From our own experience and results from simulations of light curves of bodies rotating in complex mode, coinciding with those of Samarasinha \& Mueller (2015), the most commonly detected periodicity in simulated light curves of bodies rotating in SAM corresponds to the precessional motion, that is, $P_{\phi}$, not to the total rotational period $P=2 \pi / \Omega$. If that were our case, the periodicity detected by Mottola et al. (2014), $12.4 \mathrm{~h}$, might be $P_{\phi}$. In this circumstance, as previously, the $270 \mathrm{~h}$ might correspond to the spin period, $P_{\psi}$, or to any combination of motions. We call this case 2 , and it is characterized by $P_{\phi}=12.40 \mathrm{~h}$ and $P>12.40 \mathrm{~h}$. After considering these two cases, it is still necessary to point out that from series of OSIRIS images that cover a full period of $12.40 \mathrm{~h}$, the body seems to rotate with that period, meaning that the orientation of the body in space approximately returns to its starting orientation after the $12.40 \mathrm{~h}$ period. This clearly favors case 1 , but we still kept case 2 , which is discarded if $P$, depending on the value of $P_{\psi}$, is very different from $12.40 \mathrm{~h}$.

\section{Theoretical simulations}

To study the behavior of the spin axis in an inertial frame, simulations with different inertia moments and excitation levels were performed. We calculated Euler angles and their velocities and transformed the instantaneous orientation of the spin axis to J2000 to compare it with the data shown in Fig. 2. Additionally, simulated RA/Dec coordinates were analyzed by means of the Lomb technique to determine which periodicities would be detected. Noise and averaging of theoretical data were considered to simulate actual circumstances as best possible. In the simulations, the real dependence of Euler angles with all involved variables, as described in Samarasinha \& Mueller (2015), for instance, was considered. The only simplification was that to be able to perform the calculations in a systematic and easy way, we considered that the angular momentum modulus and the rotational energy can initially be defined as $L=I_{z} \cdot \Omega$ and $E=0.5 / C \cdot I_{z}^{2} \cdot \Omega^{2}$, respectively. This approach assumes that $\Omega \approx \Omega_{z}$, neglecting the appropriate contribution to $\Omega_{x y z}$ of the components $\Omega_{x}$ and $\Omega_{y}$. This approach is a reasonable sacrifice as starting point given the expected low excitation level, in which $E_{l} \approx 0$, and $C / I_{z} \approx 1$. Evaluated a posteriori, the highest excitation level necessary to interpret the observationally derived data is on the order of $10^{-4}$. In case 1 we considered that the rotation velocity is $\Omega=2 \pi / P=2 \pi /(12.4 * 3600) \mathrm{rad} \mathrm{s}^{-1}$, with $P$ the periodicity detected by Mottola et al. (2014). For case 2 , in which the precession period $P_{\phi}$ is identified with the periodicity detected by Mottola et al. (2014), the rotational period P, and therefore the rotational energy and the modulus of the angular momentum, depend on the meaning assigned to the RA/Dec periodicity. Thus, in case $2, P$ is explicitly discussed below.

\subsection{Simulating the homogeneous body}

The orientation of the spin axis in J2000 was calculated for a body with inertia moments equal to those derived from the geometric shape of 67P assuming a homogeneous density distribution. Calculations were performed for different excitation levels $E_{l}$ by visually checking if the RA/Dec distribution of theoretical data agreed with the observed distribution (displayed in Fig. 2). This case was also considered to study the effect of averaging on the spin axis RA/Dec distribution and on the detected periodicities. The simulation for case 1 with a value of $E_{l}=1.9 \times 10^{-6}$ is shown in Fig. 5a. This figure shows that the instantaneous simulated spin axis orientation displays a pattern that may barely be considered similar to the observationally derived one. An important difference between observational and theoretical coordinates comes from the inner hole, which is clearly visible in the simulated data and missing in the actual spin axis orientation. This inner hole exists because the angle of nutation has a lowest value different from zero, mainly due to the excitation level. To decrease the size of this inner hole, the excitation level must be reduced. This would demand a modification of the inertia moments, which would imply some inhomogeneity, if we still wish to cover the observational range in RA and Dec coordinates. In addition to visual comparison, if a two-sided KolmogorovSmirnov test is applied to estimate compatibility between the observationally derived and the theoretical RA distributions, the probability of being compatible is lower than 0.05 . This probability drops to 0.02 if Dec distributions are compared. Differences and poor compatibility between observational and theoretical coordinates are at the moment expected because simulated data are instantaneous values while the observationally derived coordinates are the result of an averaging within $10 \mathrm{~h}$. Before we describe the effect of averaging on the RA/Dec distributions, we describe the detected periodicities when a time-series analysis software is applied to the RA/Dec distribution. This is to be compared with Fig. 3.

\subsubsection{Meaning of the detected periodicities}

Figure $5 b$ shows the Lomb periodogram of the simulated Dec distribution shown in Fig. 5a that is considered ideal, meaning that neither noise nor averaging were included. The periodogram of the simulated RA coordinates would be exactly the same as that of simulated Dec coordinates, with only small differences in spectral power. This figure shows that two clear periodicities are 

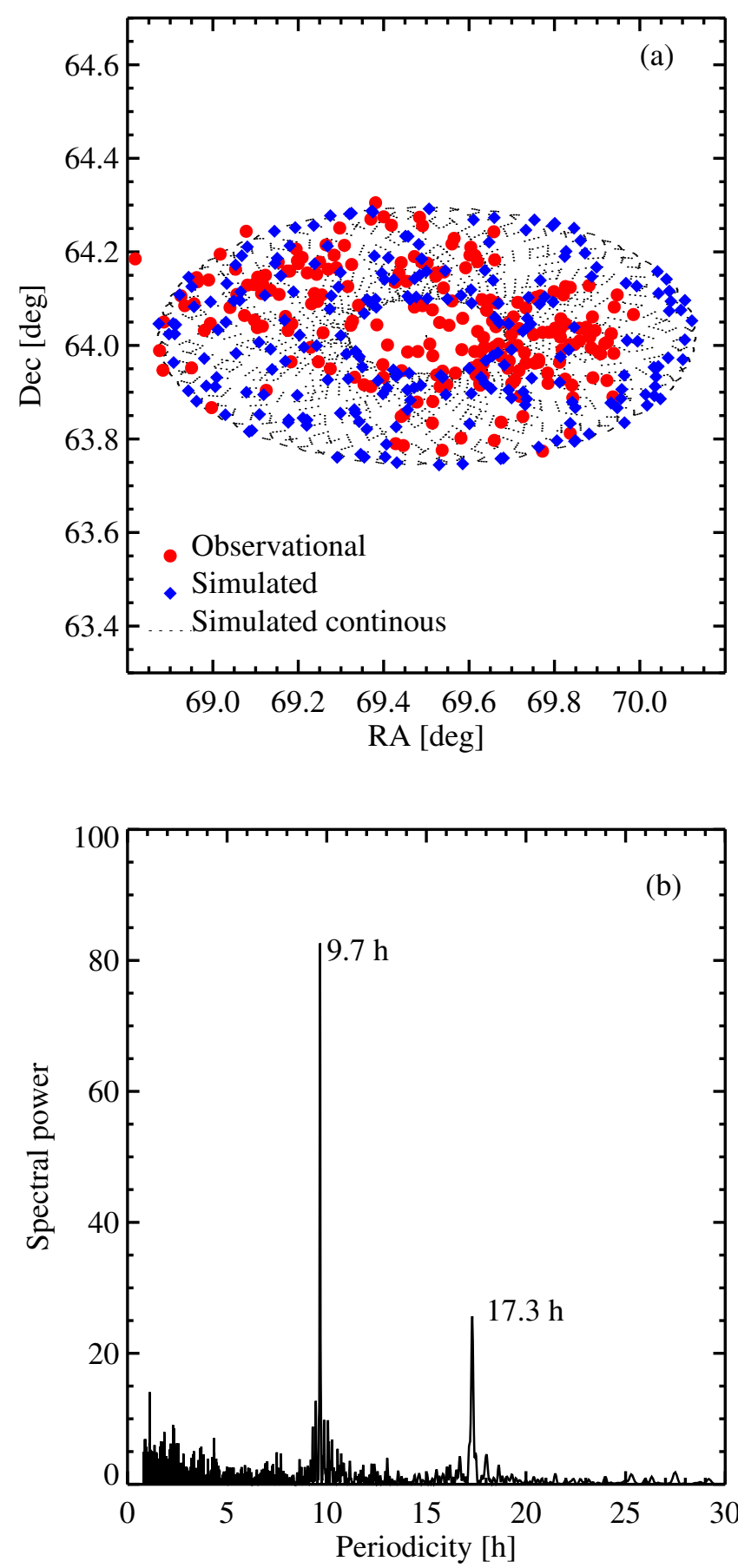

Fig. 5. a) Bullets $(\bullet)$ show the spin axis orientation as determined with the SPC method (Fig. 2). Small dots (.) are the spin vector orientation for a simulated body with inertia moments equal to those derived from the shape of $67 \mathrm{P}$ assuming homogeneity, and an $E_{l}=1.9 \times 10^{-6}$ under case 1 circumstances. These data are displayed with a time separation of $1 \mathrm{~h}$. Diamonds $(\diamond)$, to be compared with the bullet distribution, correspond to the orientation of the spin axis for the simulation performed at the time and date associated with each determination made with the SPC method. To locate the diamonds, neither noise nor averaging have been included. b) Lomb periodogram of the Dec-simulated coordinates (diamonds) shown in Fig. 5a. The RA coordinate periodogram is exactly the same as that of the Dec data, only small differences in spectral power (height of the peak) can be appreciated.

found. They are at $9.67 \mathrm{~h}$ and at $17.3 \mathrm{~h}$. From the Euler equations it is possible to estimate that, under case 1 circumstances and for the inertia moments and excitation level considered, this simulation has a $P=2 \pi / \Omega=12.4$ h while the theoretical $P_{\phi}$ and $P_{\psi}$ would be $9.66 \mathrm{~h}$ and $43.75 \mathrm{~h}$, respectively. This means that while the rotational period would be $12.4 \mathrm{~h}$, the body would be spinning with a mean period of $43.75 \mathrm{~h}$ around the third axis of inertia and that this axis, at the same time, would be precessing around the angular momentum vector with a mean precession period of $9.66 \mathrm{~h}$. The periodogram shows that the most significant detected periodicity (working with ideal data) corresponds exactly to $P_{\phi}$, the mean precession period (for the inertia moments and $E_{l}$ considered, i.e., $9.66 \mathrm{~h}$ ). The other significant periodicity corresponds to a combination $P_{\mathrm{c}}$ of $P_{\phi}$ and $P_{\psi}$, particularly to

$P_{\mathrm{c}}=\frac{1}{\frac{1}{P_{\phi}}-\frac{2}{P_{\psi}}}$,

which in our simulation is exactly $17.3 \mathrm{~h} . P_{\mathrm{c}}$ is therefore obtained as a combination of the precession period and half the spin period, which is exactly the nutation period. These two significant periodicities, $P_{\phi}$ and $P_{\mathrm{c}}$, are the only ones found in all simulations performed considering a wide range of inertia moments and excitation levels. None of the simulations showed a periodicity different from $P_{\phi}$ and/or $P_{\mathrm{c}}$ in the analysis of the simulated RA/Dec coordinates. In none of the simulations, $P_{\psi}$ or $2 \pi / \Omega$ were detected. Moreover, from the analysis of instantaneous simulated data, the most significant periodicity was always $P_{\phi}$ followed by $P_{\mathrm{c}}$.

When comparing the periodogram of the observationally derived coordinates (Fig. 3) with the obtained periodogram from simulated data (Fig. 5b), we see that they are very different from each other. This indicates, again, that the homogeneous case seems to be incompatible with our measurements, at least when averaging is not considered and under case 1 circumstances.

As for case 2 circumstances $\left(P_{\phi}=12.40 \mathrm{~h}\right)$, we recall that it is not possible to estimate the rotational energy without attributing a meaning to the $270 \mathrm{~h}$ periodicity. Nevertheless, it is possible to proceed the other way around. Considering the inertia moments of the homogeneous body, we may search for the rotational energy corresponding to a value of $P_{\phi}$ equal to $12.40 \mathrm{~h}$. If this exercise were carried out, we would find that the body spins with a period $P_{\psi} \approx 56.16 \mathrm{~h}$ and precesses with a $P_{\phi}=12.4 \mathrm{~h}$, displaying a total rotational period of $P \approx 15.9 \mathrm{~h}$. This situation would lead to a $P_{\mathrm{c}}$ of approximately $22.21 \mathrm{~h}$. None of these periodicities can be related with the RA/Dec periodicity of $270 \mathrm{~h}$. Additionally, as previously, the periodogram of simulated data only shows the periodicities corresponding to $P_{\phi}$ and $P_{\mathrm{c}}$, that is, $12.40 \mathrm{~h}$ and $22.21 \mathrm{~h}$.

\subsubsection{Averaging effect}

The observational RA/Dec coordinates derived by the SPC method include an averaging within $10 \mathrm{~h}$, which was not included in previous figures (Figs. 5a and 5b). Figures $6 \mathrm{a}$ and $6 \mathrm{~b}$ show the effect of such averaging both in the RA/Dec pattern and in the Lomb periodogram. The first evident consequence is that the averaging significantly reduces the range of the RA and Dec coordinates. This is a logical result because the averaging interval $(10 \mathrm{~h})$ is larger than the precession period $(9.66 \mathrm{~h})$. Consequently, to make the simulation compatible with observations, an excitation level higher than the one we considered is necessary. As for the Lomb periodogram (Fig. 6b), while the same two periodicities are still clearly detected $(9.67 \mathrm{~h}$ and $17.3 \mathrm{~h})$, Figs. $5 \mathrm{~b}$ and $6 \mathrm{~b}$ are different. The averaging reduces the significance of the periodicity-affected $\left(P_{\phi}\right)$, while it increases that of 

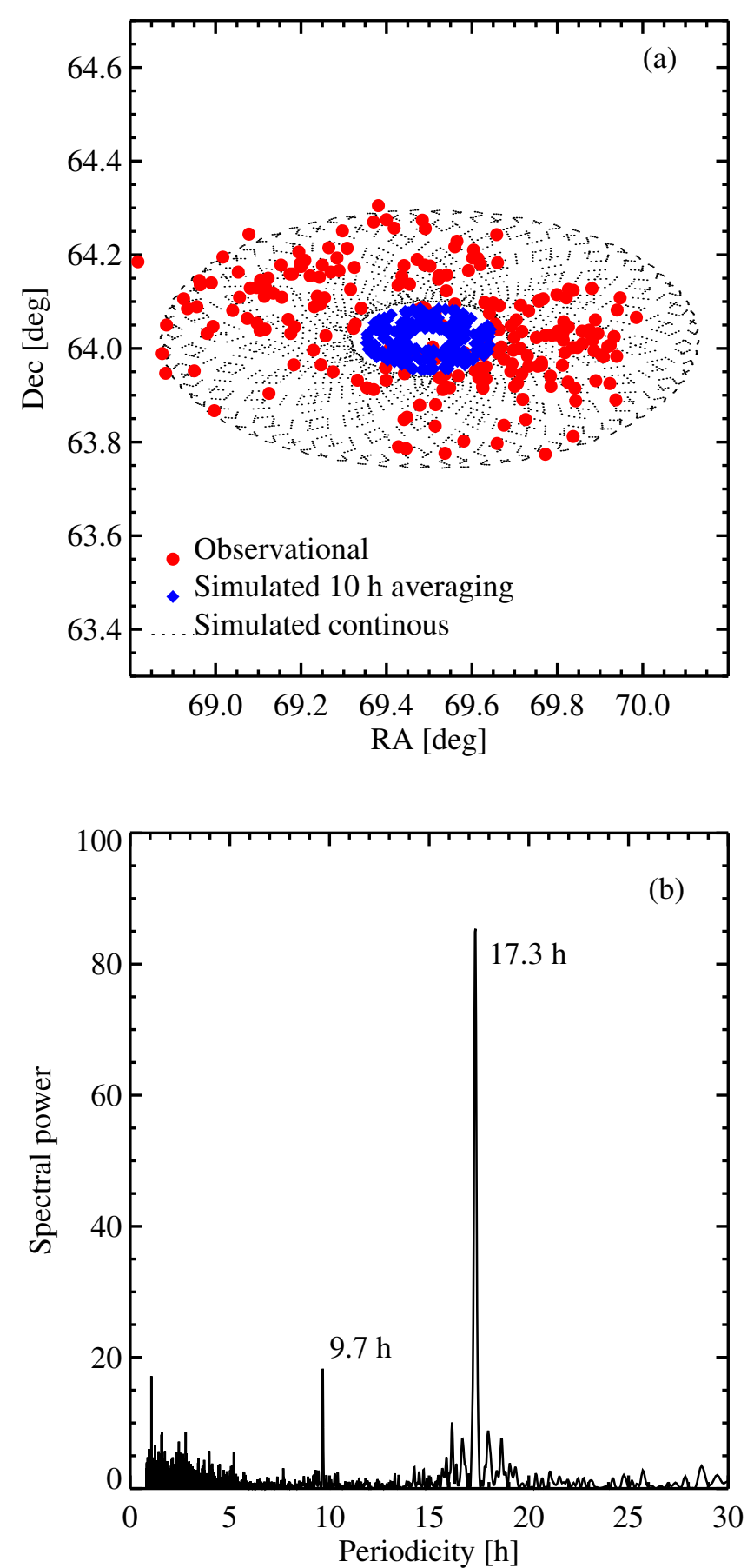

Fig. 6. a) As in Fig. 5, bullets (•) show the spin axis orientation as determined with the SPC method (Fig. 2). Small dots (.) are the spin vector orientation for a simulated body with inertia moments equal to those derived from the shape of 67P assuming homogeneity, and an $E_{l}=1.9 \times 10^{-6}$ under case 1 assumptions, that is, exactly the same circumstances as those of the simulation shown in Fig. 5a. These data are displayed with a time separation of $1 \mathrm{~h}$. Diamonds $(\bullet)$, to be compared with the bullet distribution and also with Fig. 5a, correspond to the simulated spin axis orientation at the moments associated with each determination with the SPC method, but now calculated as the average value of the instantaneous coordinates within intervals of $10 \mathrm{~h}$ around these moments. b) Lomb periodogram of the simulated averaged Dec coordinates (diamonds) shown in Fig. 6a.

$P_{\mathrm{c}}$. It could be said that the averaging "transfers" significance from $P_{\phi}$ to $P_{\mathrm{c}}$.
Table 1. $I_{z} / I_{y}$ ratios as a function of $I_{y}$ to have a $P_{\psi}$ of approximately $270 \mathrm{~h}$ when $E_{l}=3 \times 10^{-5}$ under case 1 and case 2 circumstances.

\begin{tabular}{lll}
\hline \hline$I_{y}$ & $I_{z} / I_{y}($ case 1$)$ & $I_{z} / I_{y}($ case 2$)$ \\
\hline 1.10 & 1.018 & 1.019 \\
1.20 & 1.010 & 1.011 \\
1.30 & 1.007 & 1.008 \\
1.40 & 1.005 & 1.006 \\
1.50 & 1.004 & 1.005 \\
1.60 & 1.004 & 1.004 \\
1.70 & 1.003 & 1.003 \\
1.80 & 1.003 & 1.003 \\
1.90 & 1.002 & 1.003 \\
2.00 & 1.002 & 1.002 \\
$2.10-3.0$ & $<1.002$ & $<1.002$ \\
\hline
\end{tabular}

Figure 6a clearly shows that the averaging makes the simulated RA/Dec pattern incompatible with the observational pattern. Keeping the inertia moments, the only variable available to make the two data sets more compatible would be $E_{l}$. It could be shown that an $E_{l}$ of about $3 \times 10^{-5}$ is necessary when averaging is included in theoretical data to have a $\theta_{\max }$ covering the full range of the observationally derived RA/Dec data. The problem is that when $E_{l}$ is varied to increase the value of $\theta_{\max }, \theta_{\min }$ is also significantly increased. For the case of $E_{l}=3 \times 10^{-5}$, we verified that $\theta_{\min }$, when an averaging of $10 \mathrm{~h}$ is included in the theoretical data, is on the order of $0.2^{\circ}$ for the inertia moments of the homogeneous body, that is, a quantity clearly incompatible with the observationally derived RA/Dec data. Again, to make theoretical and observationally derived results compatible, it would be necessary to modify the inertia moments with regard to those of the homogenous body in addition to increasing $E_{l}$.

\subsection{Slightly inhomogeneous case: the near prolate body}

After we discarded that $P_{\phi}$, the precession period, can be identified with the RA/Dec periodicity, the most simple possible interpretation of this observational periodicity compatible with the Euler equations is to consider that it corresponds to the period $P_{\psi}$. Equation (1) indicates that $P_{\psi}$ depends on the inertia moments, the rotational energy, and the excitation level. It can be shown that the dependence with inertia moments is stronger than that with excitation level, for example, especially if the latter is close to 1 . Therefore, by fixing the excitation level to reasonable values, it is possible to find the approximate relationship $I_{z} / I_{y}$ that corresponds to a value $P_{\psi}$ of approximately $270 \mathrm{~h}$, always assuming that $L \approx I_{z} \Omega$. Under case 1 circumstances, we recall that $\Omega=0.0014075 \mathrm{rad} \mathrm{s}^{-1}$. Under case 2 assumptions, if $\dot{\phi}=$ $0.0014075 \mathrm{rad} \mathrm{s}^{-1}$, and $\dot{\psi}=2 \pi /(270 \cdot 3600)=6.46 \times 10^{-6} \mathrm{rad} \mathrm{s}^{-1}$, it is possible to approach $\Omega \approx \Omega_{z} \approx \dot{\phi}-\dot{\psi}=0.0001343 \mathrm{rad} \mathrm{s}^{-1}$ if the excitation level is considered to be low.

Some examples of these ratios are presented in Table 1. It shows that even if it is possible to find an $I_{z} / I_{y}$ ratio for any value of $I_{y}$, the body could not be homogeneous under the aforementioned interpretation. As an example, for the excitation level considered in Table 1 and an $I_{y}$ of approximately 1.83 (the value estimated from homogeneity), $I_{z}$ would have to be 1.835 , lower than the value of 1.98 estimated when the body is considered to be homogeneous. We verified that for higher excitation levels the ratio $I_{z} / I_{y}$ necessary to obtain a value of $P_{\psi}$ equal to $270 \mathrm{~h}$ also increases, but hardly reaches $1 \%$ when $I_{y}$ is that of the homogeneous body. Therefore, if $P_{\psi}$ has to be around $270 \mathrm{~h}$, regardless 

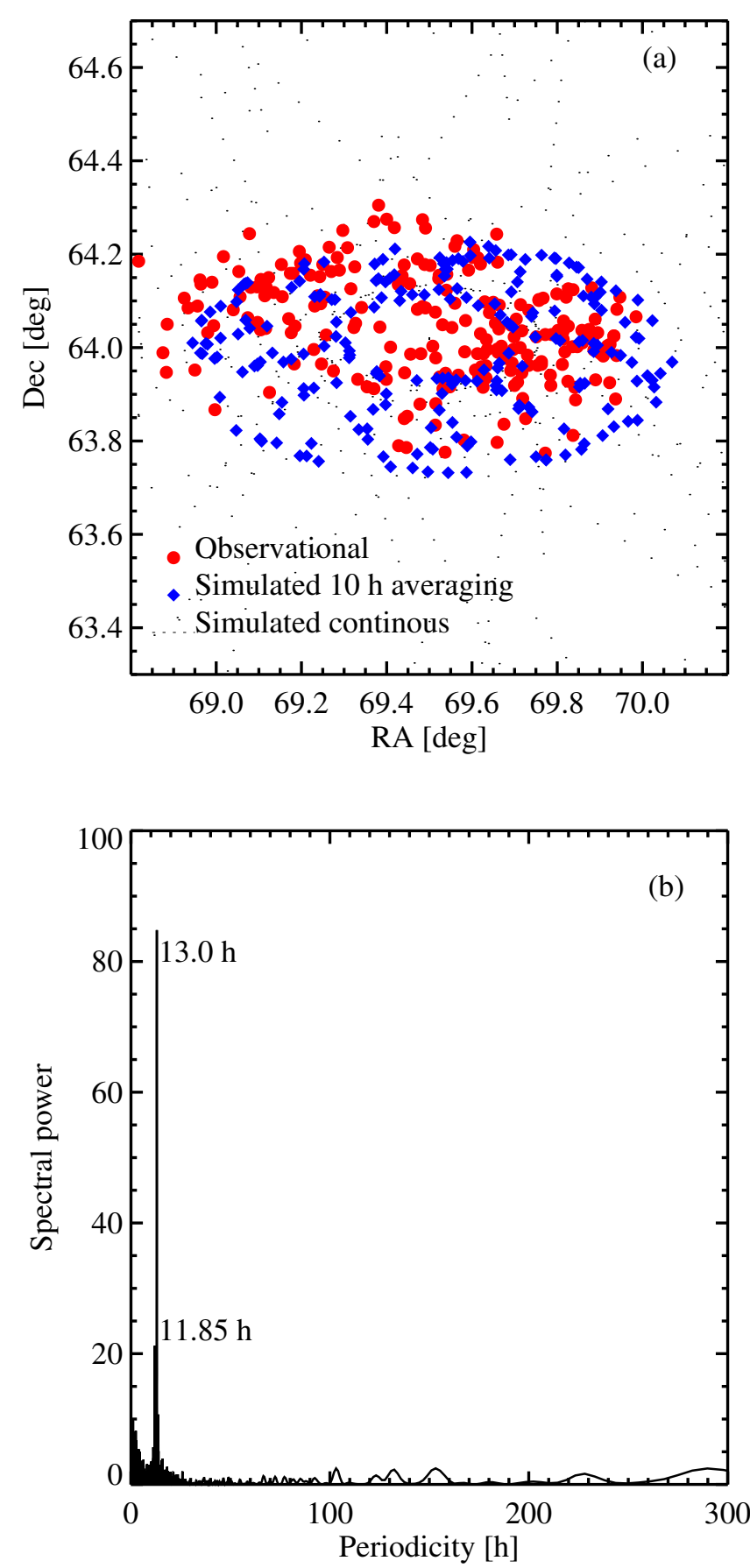

Fig. 7. a) As previously, bullets $(\bullet)$ show the observationally derived spin axis (Fig. 2). Small dots (.) are the spin axis orientation for a simulated body with inertia moments 1:1.90:1.905 and an $E_{l}=2.9 \times 10^{-6}$ (see text). The data are displayed with a time separation of $1 \mathrm{~h}$. As previously, diamonds $(\diamond)$, to be compared with the bullets distribution, correspond to the averaging within $10 \mathrm{~h}$ of the instantaneous theoretical values. b) Lomb periodogram of the Dec-simulated data (diamonds) shown in Fig. 7a. The $x$-axis here ranges up to $300 \mathrm{~h}$ to show that no significant periodicity is detected beyond $P_{\mathrm{c}}=13 \mathrm{~h}$. Given the wide range covered by the $x$-axis, the two significant periodicities $P_{\phi}=11.85 \mathrm{~h}$ and $P_{\mathrm{c}}$ appear very close in the figure.

of the interpretation of the detected $12.40 \mathrm{~h}$ periodicity (Mottola et al. 2014), the body must be slightly inhomogeneous and nearprolate (i.e., $I_{y} \approx I_{z}$ ) from the standpoint of inertia moments.

We also considered both the theoretical RA/Dec distribution and the Lomb periodograms of a body with the characteristics described above to compare them with the observationally derived ones. For illustrative purposes, a simulation under case 1 circumstances in which $I_{y}=1.90$, that is, it is increased by $4 \%$ with regard to the homogeneous situation, and $I_{z}=1.0025 \cdot I_{y}=$ 1.905 , that is, a reduction of $4 \%$ compared to the homogeneous case, is shown in Figs. 7a and 7b. The selected $I_{z}$ and $I_{y}$, which can be considered reasonable in the sense that they are not very different from the actual ones assuming homogeneity, give the ratio necessary (see Table 1 ) for $P_{\psi}$ at $268 \mathrm{~h}$ for an excitation level of $2.9 \times 10^{-6}$ (quantity selected to cover a range of RA/Dec coordinates similar to the observational one).

Figure 7a shows the theoretical RA/Dec distribution for this simulation considering an averaging of data within $10 \mathrm{~h}$, compared to the observationally derived distribution. Both distributions, simulated and real, are visually similar, although the simulated one shows a more regular pattern. A two-sided K-S test would show that theoretical and observational RA distributions are similar with a probability of approximately 0.5 . If theoretical and observational Dec distributions are compared, the probability is much lower, of approximately 0.02. Regardless of the similarity of the RA/Dec distributions, Fig. 7b shows that the Lomb periodogram of the theoretical data is quite different from the observational one (Fig. 3). For the inertia moments and excitation level considered in this simulation, we calculated that if we assume that $\Omega=0.00014075 \mathrm{rad} \mathrm{s}^{-1}$, then $P_{\phi}=11.85 \mathrm{~h}$, $P_{\psi}=268 \mathrm{~h}$, and $P_{\mathrm{c}}=13.0 \mathrm{~h}$. The periodogram shows that as previously (Figs. 5b and 6b), only two significant periodicities are detected, coinciding, again as previously, with $P_{\phi}=11.85 \mathrm{~h}$, and $P_{\mathrm{c}}=13.0 \mathrm{~h}$. The periodogram does not show any trace of periodicity at $P_{\psi}=270 \mathrm{~h}$. This test was repeated for different inertia moments and excitation levels (covering the range in Table 1) and also for case 2 circumstances, obtaining always the same results: no simulation showed a significant periodicity at $270 \mathrm{~h}$ although $P_{\psi} \approx 270 \mathrm{~h}$ in all of them. The periodograms only showed the periodicities corresponding to $P_{\phi}$ and $P_{\mathrm{c}}$.

Thus, in spite of the reasonable similarity between the simulated and observationally derived RA/Dec distributions (Fig. 7a), we find that the near prolate case is unlikely as a possible interpretation of the complex rotation of 67P. On the one hand, it is highly unlikely that such an irregular nucleus, with such a complex surface, might be near prolate from the standpoint of inertia moments. On the other hand, Lomb periodograms of theoretical data, showing just $P_{\phi}$ and $P_{\mathrm{c}}$ as detectable periodicities, do not support the interpretation that $P_{\psi}$ is at approximately $270 \mathrm{~h}$.

\section{Interpretating the RA/Dec periodicity}

A very likely interpretation of the complex rotation of 67P, supported by the Lomb periodograms of theoretical data, is that what has been detected in the periodograms of the observationally derived data (Fig. 3), that is, that the RA/Dec periodicity is actually $P_{\mathrm{c}}$ (Eq. (9)), with $P_{\phi}$ missing probably due to both averaging and errors (noise) in the determination of the RA/Dec coordinates.

It was previously mentioned that after the inertia moments are defined, the excitation level produces minor changes in the periodicities detected in the periodograms. Thus, it is possible to easily determine the $I_{z} / I_{y}$ ratio, which provides a periodicity $P_{\mathrm{c}}$ at approximately $270 \mathrm{~h}$ for cases 1 and 2 . These ratios are shown in Fig. 8, always assuming that $I_{x}$ is equal to 1 , meaning that the inertia moments are always normalized to the smallest inertia moment. As a result of the complex dependence of $P_{\mathrm{c}}$, this figure was built from a set of simulations by considering discrete values of $I_{y}$ and performing a systematic search of the $I_{z}$ defining a $P_{\mathrm{c}}$ 


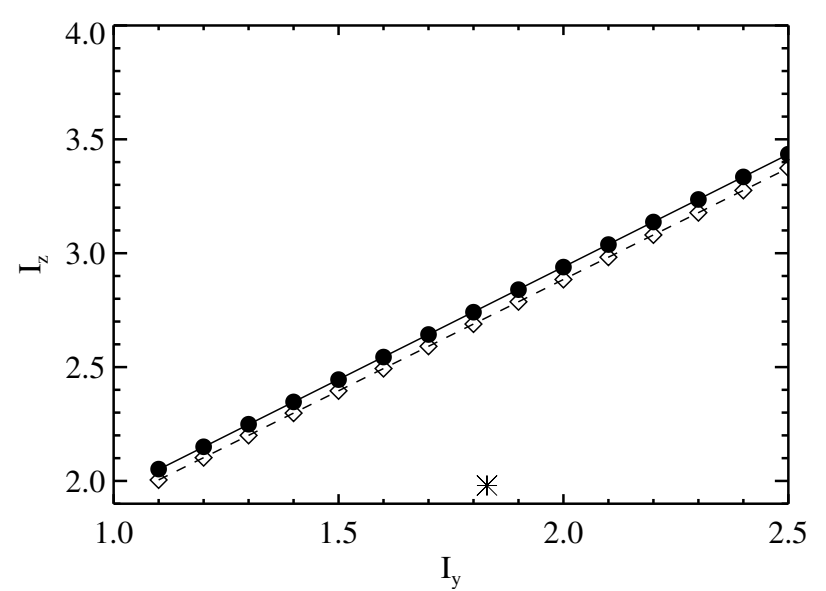

Fig. 8. Relationship between $I_{z}$ and $I_{y}$ for a $P_{\mathrm{c}}$ of approximately $270 \mathrm{~h}$ for case 1 (continuous line) and case 2 (dashed line). Both $I_{y}$ and $I_{z}$ are normalized to $I_{x}$. The asterisk (*) represents the homogeneous body.

with the desired value. A linear fit of the discrete values allows us to define the continuos relationship between $I_{z}$ and $I_{y}$,

Case 1: for $P_{\mathrm{c}}=270 \mathrm{~h}, I_{z}=0.963+0.988 \cdot I_{y}$;

Case 2: for $P_{\mathrm{c}}=270 \mathrm{~h}, I_{z}=0.929+0.978 \cdot I_{y}$.

Strictly speaking, these relationships would only be valid for the $E_{l}$ used to build it. Nevertheless, the effect on $P_{\mathrm{c}}$ of varying $E_{l}$ within reasonable values (searching for compatibility with the range of the observationally derived RA/Dec coordinates) is always smaller than the error in the periodicity detected in the periodograms of the observationally derived RA and Dec coordinates (12 h, from Jorda et al. 2016). Considering these two relationships, for cases 1 and 2 the differences are irrelevant as for the inertia moments. This accuracy in inertia moments would be very difficult to reach from any observational data given the uncertainty in cometary nature. Nevertheless, in spite of the similarity in inertia moments between cases 1 and 2, the rotational characteristics of the body depend very much on the considered case. Recalling that we always considered that $L \approx I_{z} \Omega$, under case 1 assumptions, a body with an $I_{z}$ given by the first row of Eq. (10) would have $P \approx 12.40 \mathrm{~h}, P_{\phi} \approx 6.35 \mathrm{~h}, P_{\psi} \approx 13 \mathrm{~h}$, and $P_{\mathrm{c}} \approx 270 \mathrm{~h}$, with small variations of these values depending on the excitation level (assumed to be small). This means that the body would be rotating with a period of approximately $12.40 \mathrm{~h}$, with a complex rotational motion, spinning around its principal axis with the highest inertia moment $(z)$ with a period of approximately $13 \mathrm{~h}$, while this axis precesses around the angular momentum vector with a period of approximately $6.35 \mathrm{~h}$. Under case 2 circumstances, a body with $I_{z}$ given by the second row of Eq. (10) would have $P \approx 23.71 \mathrm{~h}, P_{\phi} \approx 12.40 \mathrm{~h}$, $P_{\psi} \approx 26 \mathrm{~h}$, and $P_{\mathrm{c}} \approx 270 \mathrm{~h}$. A body like this would be spinning around the third axis of inertia with a period of $26 \mathrm{~h}$ while precessing around the angular momentum with a period of $12.40 \mathrm{~h}$, the rotational period being approximately $23.7 \mathrm{~h}$. This fact practically rules out case 2 . Under these circumstances, the body as seen from space would reach a similar orientation approximately every $24 \mathrm{~h}$, which is incompatible with the images obtained with OSIRIS. Therefore, case 2 was not considered below, except for some last comments.

Under the present interpretation (that what was detected in the periodograms of observationally derived data is $P_{\mathrm{c}}$ ), the previous relations would indicate that $67 \mathrm{P}$ cannot be homogeneous. Equation (10), for case 1, tell us that to have a period $P_{\mathrm{c}}$ around
$270 \mathrm{~h}, I_{z}-I_{y} \approx 0.96$, a quantity much larger than the corresponding difference assuming that the body has a homogeneous density, $I_{z}-I_{y}=1.98-1.83=0.15$.

In other words, while the homogeneous situation implies a ratio $I_{z} / I_{y}=1.08$, if the RA/Dec periodicity is $P_{\mathrm{c}}$, the ratio $I_{z} / I_{y}$ would be higher, ranging, for example, between 1.4 for $I_{y}=2.5$ and 1.6 for $I_{y}=1.5$ for case 1 circumstances. Except for inhomogeneity, always assuming that our starting hypothesis are valid, nothing else can be said in principle about the internal mass distribution. Inertia moments $I_{y}$ and $I_{z}$ are estimated from the integrals of the mass with the distance across the $x z$ and $y x$ planes, respectively. This means that the two principal inertia moments share the effect of density along the $x$-axis, nearly parallel to the largest geometric axis of $67 \mathrm{P}$. If the ratio $I_{z} / I_{y}$ is higher than that of the homogeneous case, the density along the $z$-axis might be lower and/or that the density along the $y$-axis might be higher than in the homogeneous case. Interestingly, $z$-axis goes across the neck of 67P, a region certainly peculiar even in the already quite odd surface of 67P. In any case, we understand that the previous $I_{z}-I_{y}$ difference can be considered quite extreme. The equivalent ellipsoid would tend to be oblate, with an intermediate-to-short axes ratio of approximately 7 . This difference in inertia moments would therefore request a comparatively high increase of mass especially along the $y$-axis, which is practically impossible to reach with smooth and continuous variations of density across the body. For example, and in principle, the inertia moments ratio found in this study would not be compatible with a comparatively small difference in densities of the two lobes (Rickman et al. 2015; Jorda et al. 2016). One physical possibility to explain the estimated inertia moments could be a body with mass concentrated at the equatorial plane and at the surface, with a significant density variation. Another physical possibility to explain the inertia moments, again speculating with the internal structure of 67P, might be a body with comparatively small and randomly distributed holes in its interior, with mean sizes on the order of $100 \mathrm{~m}$. This latter picture would point to the heterogeneity suggested by Vincent et al. (2015). These authors concluded from their analysis of the size and spatial distribution of pits that larger heterogeneities exist in the properties of the first few hundred meters below the current nucleus surface of 67P. The possibilities given above are just some examples among many others that need appropriate modeling to be confirmed or rejected. Without constraining the ratio of inertia moments, any attempt to find a possible explanation would require additional constraints from independent measurements and/or additional hypotheses on the internal structure of $67 \mathrm{P}$ in addition to the appropriate modeling.

In addition to Vincent et al. (2015), at least certain heterogeneity in $67 \mathrm{P}$ has previously been suggested so far by Jorda et al. (2016), who detected an offset between the center of mass of the body shape assuming homogeneity and the origin of the Cheops frame. This offset suggests some heterogeneity. The modeling to interpret the inertia moments must also account for this offset. Luspay-Kuti et al. (2015) also pointed out that the compositional heterogeneity detected in the coma of 67P might suggest chemical heterogeneity in the southern hemisphere of the nucleus. Analysis of the measurements performed by the CONSERT instrument may shed some light on the internal mass distribution. Although it is still premature to draw definite conclusions because of the complexity of analyzing CONSERT data, last results points out some inhomogeneity, at least for the small lobe of 67P. Ciarletti et al. (2015) explained CONSERT measurements as due to a decrease with depth of the dielectric constant. This was interpreted by the authors as 

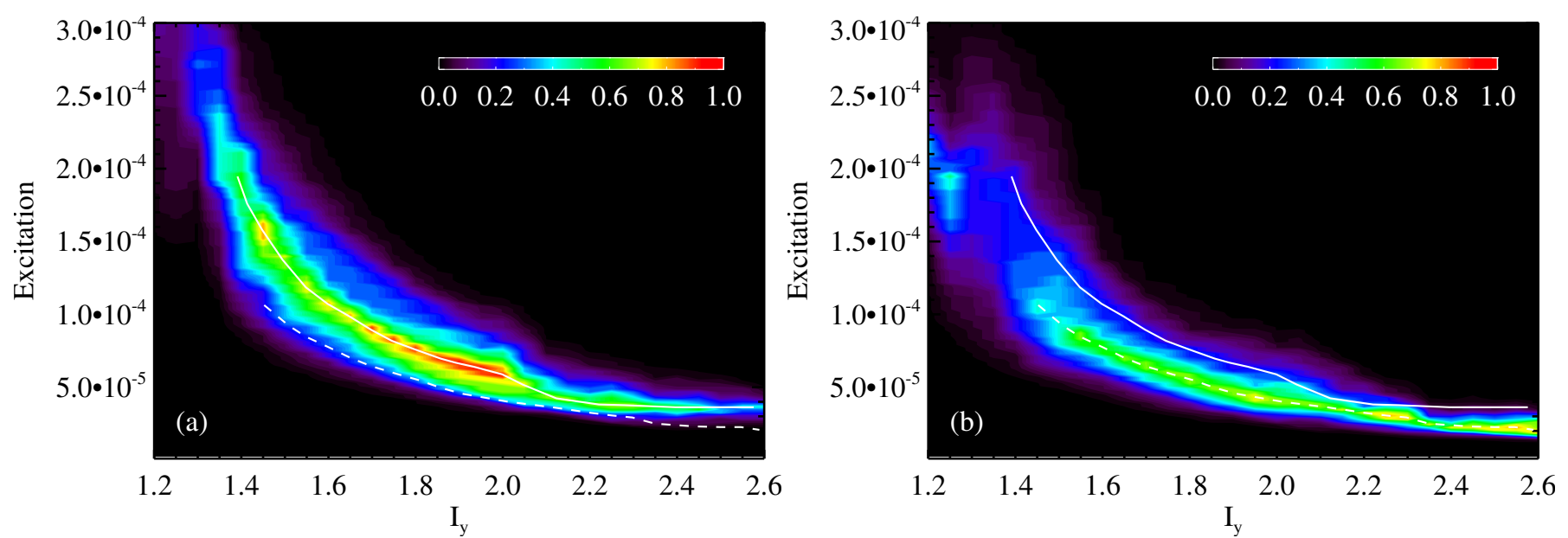

Fig. 9. a) K-S probability as a function of $I_{y}$ and $E_{l}$ when the theoretical spin axis RA distribution is compared to the observationally derived one for case 1. b) The same as a), but in this case the theoretical and observationally derived Dec distributions are compared. In the two figures, the continuous white line, $E_{l, \mathrm{RA}}=E_{l}\left[I_{y}\right]$, indicates the excitation level for each $I_{y}$ associated with the highest K-S probability when RA distributions are compared. The dashed white line, $E_{l, \text { Dec }}=E_{l}\left[I_{y}\right]$, represents the excitation level for each $I_{y}$ associated with the highest K-S probability when the Dec distributions are compared.

a possible increase of porosity with depth and/or a possible decrease of the dust-to-ice ratio. Both interpretations could point to an increase in the inertia moments in comparison with those of a homogeneous body and to a possible increase of the inertia moment ratio. During the revision process, Pätzold et al. (2016) presented their results on the gravitational field of 67P as determined from the Radio Science Instrument (RSI). According to the authors, these measurement seem to point to a homogeneous nucleus, ruling out large caverns in the nucleus interior. Nevertheless, the spatial scale of the ruled-out caverns is not clear yet, and we need to wait for the future controlled impact of the Rosetta orbiter with the surface nucleus in September 2016. At this moment, the complex gravity field could reveal details on the internal structure on the scale of hundred of meters.

The internal structure and its possible homogeneity are key characteristics for understanding cometary nuclei formation. Inhomogeneity has been suggested for several cometary nuclei as derived from different sources. For example, Kawakita et al. (1997) concluded that the presumable parent of comets C/1996 Q1 (Tabur) and C/1988A1 (Liller) was inhomogeneous because the gas-to-dust ratio measured in the two fragments was very different. Gibb et al. (2007) also suggested cometary heterogeneity from a compositional analysis of fragments of comet C/2001 A2 (LINEAR). There is other possible evidence of heterogeneity from other fragmenting comets, although, as pointed out by Dello Russo et al. (2007), no confident conclusion can be drawn. Chemical inhomogeneities related to possible formation mechanisms were also suggested for comets 8P/Tuttle (Bonev et al. 2008), 9P/Tempel 1 (Feaga et al. 2007), and 103P/Hartley 2 (A'Hearn et al. 2011), although evolutionary circumstances (mainly seasonal effects) cannot be ruled out (see, e.g., A'Hearn 2011).

As expected given the cometary diversity, there are also arguments favoring homogeneity in cometary nuclei. From high-quality spectra, Dello Russo et al. (2007) found that relative abundances measured from fragments $\mathrm{B}$ and $\mathrm{C}$ of comet 73P/Schwassmann-Wachmann 3 were remarkably similar, which suggests chemical homogeneity of the nucleus of 73P. We would like to stress again that discussing the heterogeneity in $67 \mathrm{P}$ is still very premature. More certain conclusions need from dedicated calculations and a more complete analysis of the internal structure of 67P by CONSERT and RSI data. If finally the suggested $I_{z}-I_{y}$ is much higher than the actual one, it would be necessary to revisit our starting hypothesis, mainly rigid-body and free-torque conditions. At the end of the paper, we briefly comment on the first of these assumptions.

\subsection{Is it possible to constrain the inertia moment ratio?}

After we defined the relation between $I_{y}$ and $I_{z}$ to have $P_{\mathrm{c}}$ on the order of $270 \mathrm{~h}$, we performed a full set of simulations for case 1 , considering as free parameters both $I_{y}$ and $E_{l}$, and fixing $I_{z}$ according to Eq. (10). In the simulations, $I_{y}$ ranged between 1.2 and 2.6, which is a reasonable interval, while a range between $3 \times 10^{-4}$ and $2 \times 10^{-6}$ was explored for $E_{l}$. This latter interval was defined after a trial-and-error procedure in which we searched for visual compatibility between observations and simulations. The results from these simulations were compared with the observationally derived data, in particular the RA/Dec pattern and the Lomb periodograms. Considering the periodograms, we verified that all simulations performed under case 1 circumstances have the most significant peak located at $P_{\mathrm{c}} \approx 270 \mathrm{~h}$ followed by a second peak located at $P_{\phi} \approx 6.35 \mathrm{~h}$. As for the RA/Dec patterns, a two-sided Kolmogorov-Smirnov test was performed to statistically evaluate the significance of the similarity between the observationally derived and the theoretical distributions. The two-sided K-S test was performed separately over RA and Dec distributions.

The similarity between the RA theoretical distribution and the observationally derived RA pattern, understood as the probability that both patterns belong to the same distribution as determined from the two-sided K-S statistic as a function of both $I_{y}$ and $E_{l}$, is shown in Fig. 9a for case 1. From the K-S statistics of the RA patterns, the region with $I_{y}$ between 1.8 and 2.0 can be clearly identified with the highest probability. The best fit, identified as the highest probability, 0.94, is obtained for $I_{y}=1.90, I_{z}=2.84$ and $E_{l}=6.7 \times 10^{-5}$. This body would be rotating with $P \approx 12.4 \mathrm{~h}, P_{\phi}=6.34 \mathrm{~h}, P_{\psi}=12.99 \mathrm{~h}$, and $P_{\mathrm{c}}=273 \mathrm{~h}$. The simulated spin axis orientation and corresponding Lomb periodograms for these parameters are shown in Figs. 10a and 10b. Considering Fig. 10a, it may be accepted that theoretical spin axis coordinates have a distribution similar to the observationally derived one. For this distribution, in 

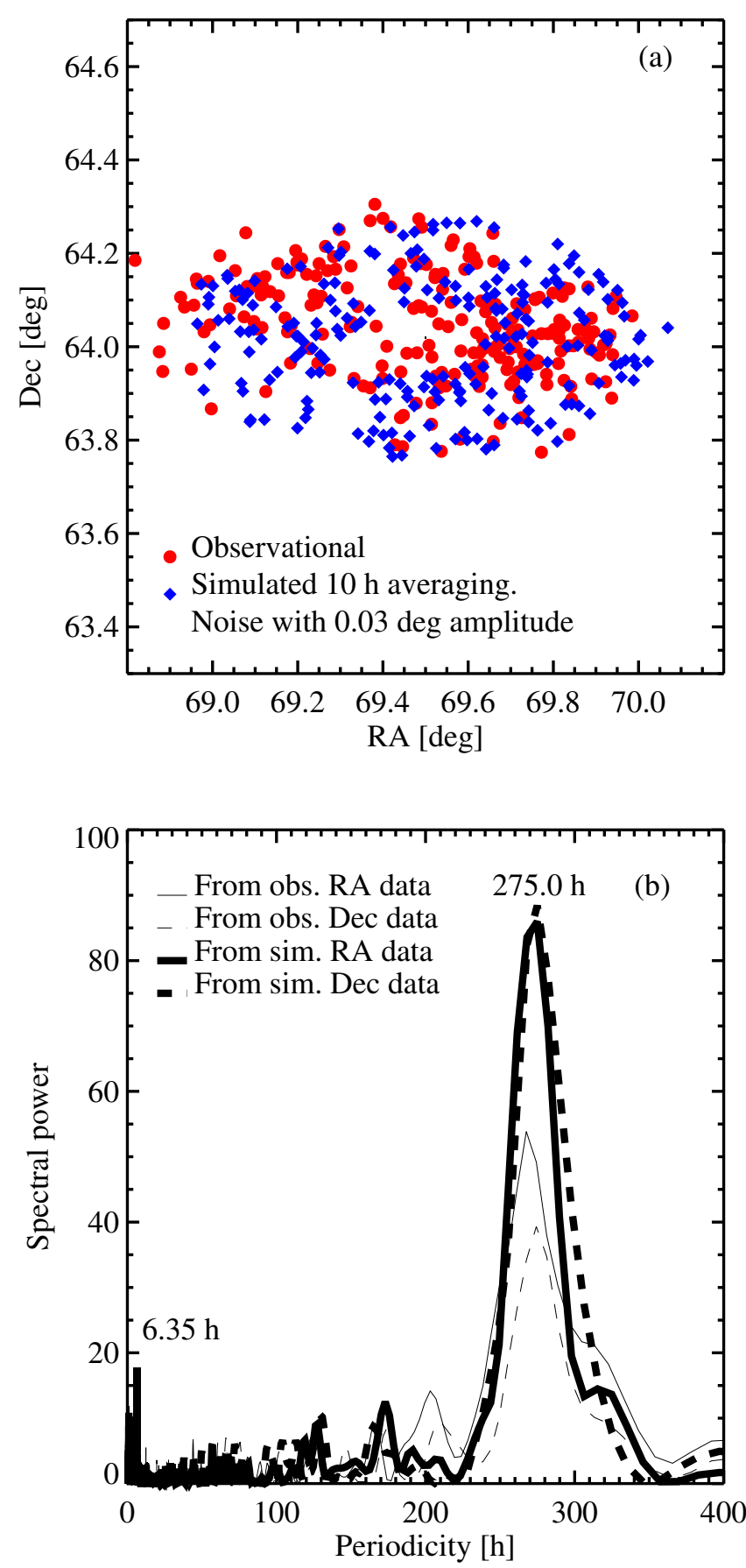

Fig. 10. Simulation with the highest K-S probability from the comparison of RA coordinate distributions. a) As previously, bullets $(\bullet)$ show the observationally derived spin axis (Fig. 2). Diamonds ( ) correspond to the simulated spin axis orientation of a body with 1:1.90:2.84, and $E_{l}=6.7 \times 10^{-5}$ when an averaging of $10 \mathrm{~h}$ and a uniformly distributed random noise with an amplitude of $0.03^{\circ}$ have been considered. b) (Thick lines) Lomb periodograms of the RA and Dec simulated data (diamonds shown in Fig. 10a). The Lomb periodograms of the observationally derived coordinates (Fig. 3) have also been included (with thin lines).

which a uniformly distributed noise with an amplitude of $0.03^{\circ}$ was included, the Lomb periodograms of the simulated RA and Dec coordinates show that the most significant peak is located at a periodicity of $275 \mathrm{~h}$, identifiable with $P_{\mathrm{c}}$, but with a spectral power significantly higher than the observational one. In addition, the periodicity corresponding to $P_{\phi}=6.35 \mathrm{~h}$ can clearly
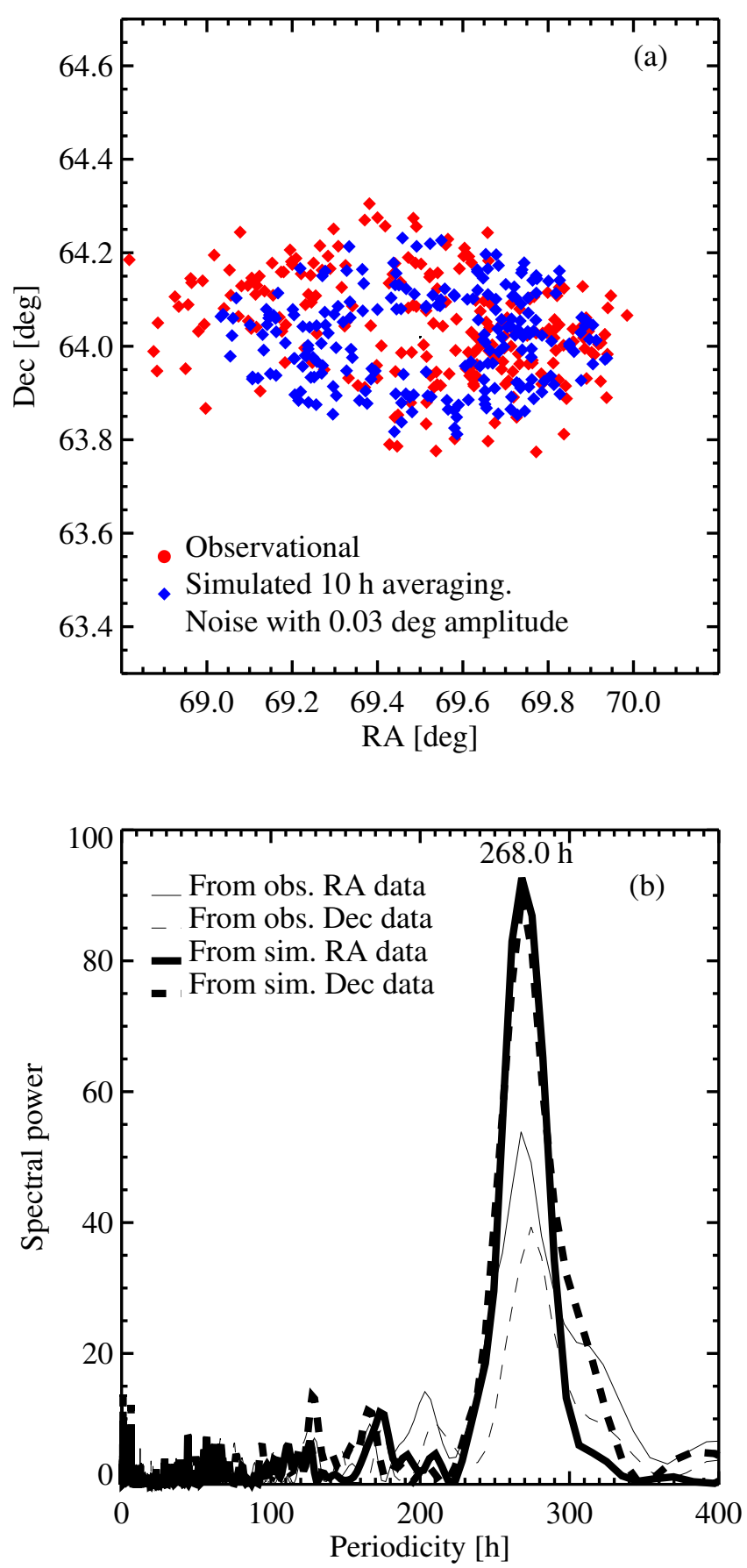

Fig. 11. Simulation with the highest K-S probability from the comparison of Dec coordinate distributions. a) As previously, bullets $(\bullet)$ show the observationally derived spin axis (Fig. 2). Diamonds ( ) correspond to the simulated spin axis orientation of a body with 1:2.25:3.19, and $E_{l}=3.1 \times 10^{-5}$ when an averaging of $10 \mathrm{~h}$ and a uniformly distributed random noise with an amplitude of $0.03^{\circ}$ have been considered. b) (Thick lines) Lomb periodograms of the RA and Dec simulated data (diamonds shown in Fig. 11a). The Lomb periodograms of the observationally derived coordinates (Fig. 3) have also been included (with thin lines).

be identified in simulated data, even though noise and an averaging significantly larger than $P_{\phi}$ were added. In principle, the differences between simulation and observations might arise because actual errors in observations are larger than formal errors. If this were true, adding a larger noise to the simulated data could erase the $P_{\phi}$ signature and also reduce the spectral power of $P_{\mathrm{c}}$. 


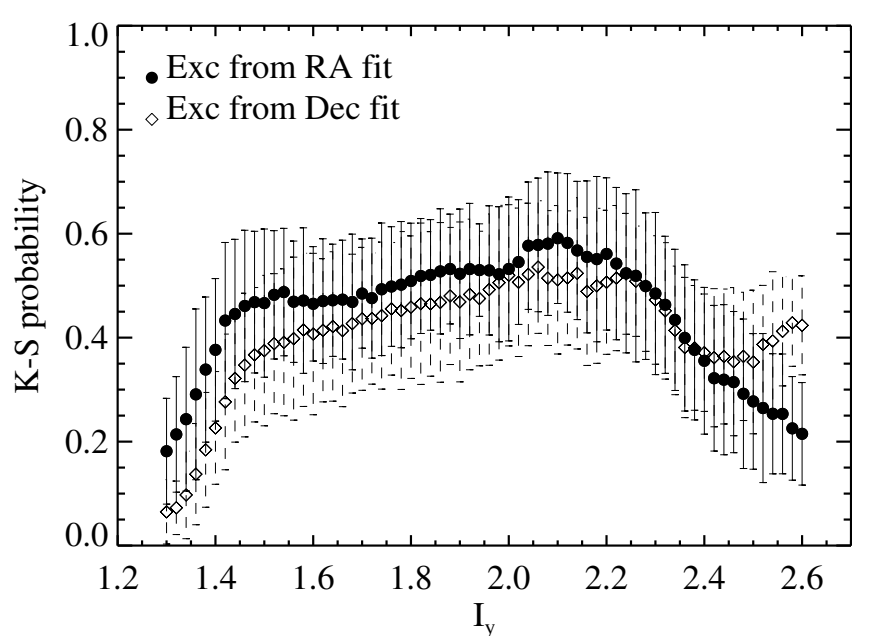

Fig. 12. K-S probability estimated as the mean of the RA and Dec probabilities for the two excitation curves, $E_{l}=E_{l}\left[I_{y}\right]$, leading to the highest probability of the K-S tests performed on RA $(\bullet)$ and Dec $(\diamond)$ data separately.

Nevertheless, in this case, the K-S tests and the highest probability case would not be valid anymore because it would be necessary to at least modify $E_{l}$ to adjust the range of the observationally derived RA/Dec.

The K-S probability that compares the observationally derived Dec distribution with the theoretical one as a function of $I_{y}$ and $E_{l}$ is shown in Fig. 9b for case 1. This figure shows that several regions with comparatively high probabilities can be identified, with a highest value of 0.82 . Even though it is smaller than the value obtained from the RA comparison, this value could be considered to have a similar significance because it was estimated that the dispersion in the probability due to the observational error is on the order of 0.1 . The K-S probabilities within that range may therefore be considered to have similar significance. From the Dec comparison, the highest probability is obtained for the combination $I_{y}=2.25, I_{z}=3.19$, and $E_{l}=3.1 \times 10^{-5}$. As previously, and as in all simulations under case 1 circumstances, this body would also be rotating with $P \approx 12.4 \mathrm{~h}, P_{\phi}=6.35 \mathrm{~h}, P_{\psi}=13.0 \mathrm{~h}$, and $P_{\mathrm{c}}=271 \mathrm{~h}$. The simulated spin axis orientation and corresponding Lomb periodograms for these parameters are shown in Figs. 11a and 11b. The comparison between simulated and observationally derived spin axis coordinates show that the discrepancies are noticeable here, especially in the RA range. Nevertheless, the Lomb periodogram still shows the most significant periodicity close to $P_{\mathrm{c}}$, at $268 \mathrm{~h}$, with very weak traces of $P_{\phi}$. We note from simulations not shown that in addition to noise and averaging, the value of $I_{y}$ also affects the significance (i.e., height of the peak in Lomb periodograms) of $P_{\phi}$. For values higher than $I_{y} \approx 2.0$, the peak corresponding to $P_{\phi}$ is hardly detectable. As for $P_{\mathrm{c}}$, the spectral power of simulated data is much higher, as in the RA case, than that of the observationally derived ones. Again, a larger actual error in observations might conciliate both spin axis RA/Dec distribution and the spectral power of $P_{\mathrm{c}}$, but it would be necessary to recalculate the K-S probability.

Bearing in mind that the dispersion introduced by the error in RA/Dec in the K-S probability is on the order of 0.1, we cannot favor any set of parameters. An additional statistical test was performed to determine whether it was possible to find a best common region for RA and Dec coordinates together. For each $I_{y}$, the inertia moment $I_{z}$ was calculated by using Eq. (10), and

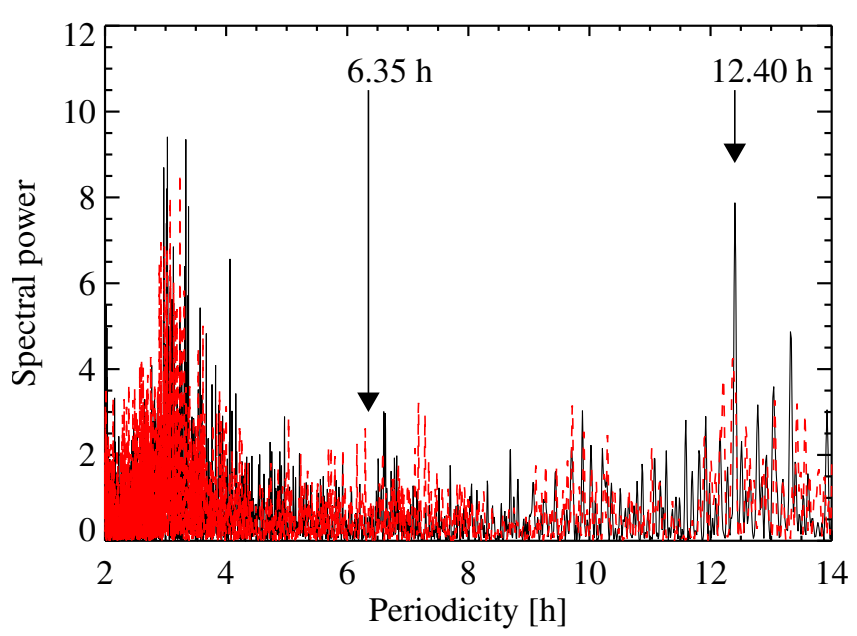

Fig. 13. Lomb periodograms of the observationally derived RA (black) and Dec (Red) coordinates (Fig. 3) for periodicities lower than $14 \mathrm{~h}$. Arrows indicate the position of $P_{\phi}$ for the two possibilities discussed in the text, $6.35 \mathrm{~h}$ and $12.40 \mathrm{~h}$.

first $E_{l}$ was defined by using the continouos white line shown in Figs. 9a and b.This white line defines the highest probability as a function of $I_{y}$ when RA coordinates are compared. After we defined the set of $\left[I_{y}, I_{z}\right.$ and $\left.E_{l}\right]$, the theoretically obtained RA and Dec coordinates were perturbed with random and uniform noise with an amplitude of $0.03^{\circ}$. One hundred perturbed RA and Dec patterns were built for each $\left[I_{y}, I_{z}\right.$ and $\left.E_{l}\right]$ combination. The K-S probabilities for all these patterns were calculated by comparing them with the observationally derived RA and Dec distributions, building the final K-S probability as ([K$\left.\mathrm{S}]_{\mathrm{RA}}+[\mathrm{K}-\mathrm{S}]_{\text {Dec }}\right) / 2$. We then repeated the calculations by using the dashed white line shown in Figs. 9a and 9b, that is, the excitation level corresponding to the highest K-S probability for each $I_{y}$ when simulated and observationally derived Dec coordinates were compared. The results, in terms of median and standard deviation of the 100 different random patterns, are shown in Fig. 12. This figure shows that when the RA and Dec K-S probability is averaged and random low-amplitude noise is added, there is no preferred combination of $\left[I_{y}, I_{z}\right]$ for any of the two excitation curves that best fit the RA and Dec coordinates separately. Figure 12 shows that any $I_{y}$ within the range $1.5-2.3$ is virtually similar in terms of K-S probability when RA and Dec coordinates are considered together. Therefore, no $\left[I_{y}, I_{z}\right.$ and $\left.E_{l}\right]$ combination can be defined from the K-S tests.

\subsection{Is there any trace of $P_{\phi}$ in the periodogram of observationally derived data?}

We showed that simulations indicate that $P_{\phi}$, the precessional period, may be detectable in the periodograms even when an averaging period longer than $P_{\phi}$ is used when retrieving RA/Dec coordinates. The reason might be that the $\phi$ angle is not strictly periodic and that $P_{\phi}$ is defined just from the mean value of the instantaneous $\dot{\phi}$. Therefore, even when the averaging is large, some signatures of $\dot{\phi}$ may remain in the space position of the spin axis. We therefore explored the periodogram of RA/Dec coordinates in the region of periods shorter than $14 \mathrm{~h}$ to determine whether there was any trace of the $6.35 \mathrm{~h}$ periodicity. The relevant region of the periodogram of the observationally derived $\mathrm{RA} / \mathrm{Dec}$ coordinates is shown in Fig. 13. This figure shows that neither RA nor Dec periodograms show any signature at $6.35 \mathrm{~h}$. Nevertheless, the RA coordinates periodogram displays a weak 
signature at $12.40 \mathrm{~h}$ that is also present in the Dec coordinates periodogram. If our interpretation that the highest peak in the periodogram is $P_{\mathrm{c}}$ with some potential signatures of $P_{\phi}$ is correct, this weak signature would point to case 2 circumstances. Nevertheless, as stated above, this would imply that $67 \mathrm{P}$ spins around the axis with the highest inertia moment with a period of $26 \mathrm{~h}$ while this axis precesses around the angular momentum vector with a period of $12.40 \mathrm{~h}$, with $P_{\mathrm{c}} \approx 270 \mathrm{~h}$ and the total rotational period is approximately $23.7 \mathrm{~h}$. This solution would require reinterpreting the periodicity detected by Mottola et al. (2014) as the precession period, but more importantly, it would not be compatible with OSIRIS images. In principle, the signature at $12.40 \mathrm{~h}$ is really weak with a significance level of $3 \%$, and therefore could be spurious. Nevertheless, its coincidence with the periodicity detected by Mottola et al. (2014) indicates that it might be real. In that case, its presence is unexpected, and based on the information obtained from theoretical periodograms, there is currently no satisfactory explanation, except if it is a coincidence.

\subsection{Comments on the rigid-body assumption}

The previous analysis and discussions were performed under the assumption that the body is considered rigid. In principle, this assumption is not necessarily true and it might be that non-rigidity is the cause of the motion of the spin axis described in Fig. 2, or at least of part of it. It is beyond the scope of this paper to study the sources of non-rigidity in depth as a possible cause of the RA/Dec periodicity, but we consider the potential effects of the most likely ones below.

A body rotating in complex mode, that is, on an excited level, dissipates rotational energy mostly as heat as a result of the friction of small and slow internal mass displacements. Because this is an internal adjustment, the angular momentum does not change, which means that the small displacements are translated into changes of the rotational velocity vector, inertia moments, and mass center. We know that the rotational period $(2 \pi / \Omega)$ or the precessional period $\left(P_{\phi}\right)$, depending on the interpretation, did not change within a very small error during the time covered by the observations used in this study. This would imply that inertia moments did not change and that therefore any motion of the spin axis that is due to some potential damping of the excitation energy is safely discarded. This agrees with the estimate of the timescale necessary to damp an excited rotational state by internal dissipation as derived by Burns \& Safronov (1973) and applied to some comets by Jorda \& Gutiérrez (2000). These latter authors showed that by using typical values for the parameters involved in the timescale, it may range from a few thousand of years (for large nuclei) to up to $10^{7}$ years (for small nuclei), which is considerably longer than the period of time covered by our observations. Other sources of non-rigidity such as erosion by activity and deposition of the heaviest particles falling back onto the surface that also induce changes in the inertia moments (and therefore in the rotational velocity) have equally very long timescales (see, e.g., Jorda \& Gutiérrez 2000).

Related to non-rigidity, one possibility that immediately comes to mind is whether the comet nucleus may be suffering a kind of Chandler wobble. Before considering this possibility, we describe this motion including the Euler angles. The Chandler wobble is generally described as the torqueless precession of the Earth with a period of about 433 days. This means that the motion of the axis of rotation ( $\boldsymbol{\Omega}$, North Pole at Earth) around the axis of symmetry of Earth $(z)$ which is assumed to be an oblate body. It is important not to confuse this precession with the term precession as used throughout this study, meaning the motion of the $z$-axis around the angular momentum. This may lead to some confusion with the periodicities. In our case, as we described, the mean precession period would always be $P_{\phi}$. If the motion of the rotation vector around the third axis of inertia is considered, the former precesses around the axis with the highest inertia moment $(z)$ exactly with the period $P_{\psi}$, that is, our spin period. On the one hand, Earth, which is assumed to be an oblate body, does not fulfill the Euler equations and the period of $\boldsymbol{\Omega}$ around the axis of symmetry is longer than expected from Euler equations for the known inertia moments. On the other hand, it has been calculated that the Chandler wobble would be damped in less than 100 years, unless some internal force were causing it. A clear explanation for the Chandler wobble is still lacking, but it might be related to fluctuating pressure at the bottom of the oceans, caused by temperature, salinity, and circulation changes (Gross 2000), or even to centrifugal deformation of the portion of the Earth's mass contained in circulating fluids (Jenkins 2015). Regardless of the final explanation, it seems that Chandler wobble is due to the presence of liquid (or, in general, movable) mass that may deform and/or act on the rest of the body. Interestingly, the deformability of Earth may be estimated as (see, e.g., Jenkins 2015)

$k=1-\frac{\omega_{\text {Chandler }}}{\omega_{\text {Euler }}}$,

where $\omega_{\text {Chandler }}$ is the frequency associated with the Chandler wobble and $\omega_{\text {Euler }}$ is the frequency of the precession of the rotation axis around the axis with the highest inertia moment. According to the Euler equations for the inertia moments of Earth, $\omega_{\text {Euler }}$ is estimated to be $2 \pi / P_{\psi}=2 \pi / 306$ days $^{-1}$. If the corresponding figures are used, $k$ of Earth is estimated to be 0.293 , which is fully consistent with the Love number $k_{2}$ computed form the magnitudes of terrestrial and oceanic tides (see, e.g., Stacey \& Davis 2008).

Assuming that in our case $\boldsymbol{\Omega}$ were precessing around the axis with the highest inertia moment and that for some reason it translates its periodicity into the detected RA/Dec periodicity, we could also estimate the deformability necessary by using the previous expression. Assuming homogeneity, that is, that the inertia moments as derived from the body shape if density is assumed to be homogeneous, the precession period of $\boldsymbol{\Omega}$ around the axis with the highest inertia moment would be $P_{\psi \text { Euler }}=43.75 \mathrm{~h}$ for case 1 . If our body were suffering some type of Chandler wobble with a $P_{\text {Chandler }}=270 \mathrm{~h}$ (i.e., the RA/Dec periodicity), then the deformability could be estimated as 0.838 . This would represent an unreasonably high number if deformability, as for Earth, is identified with the Love number $k_{2}$.

The previous discussion does not totally discard that body deformation, or non-rigidity in general, is the cause of the RA/Dec periodicity detected from observations. An in-depth study is beyond the scope of this paper, which is based on the rigid-body assumption. We merely wished to illustrate that the most intuitive possibilities related to non-rigidity are ruled out.

\section{Summary and conclusions}

Spin axis orientation data as obtained during the SPC procedure to retrieve the shape of $67 \mathrm{P}$ show evidence that its nucleus was rotating in complex mode. This evidence is compatible with and confirms the early detection of possible complex rotation by Preusker et al. (2015). When the spin axis coordinates were studied by means of different time-series analysis techniques, the periodograms showed a very significant periodicity at approximately $270 \mathrm{~h}$, different from the periodicity determined by 
Table 2. Summary of the main results obtained from the simulations performed to interpret the periodicity of approximately $270 \mathrm{~h}$ detected from the periodograms of the coordinates of the spin axis.

\begin{tabular}{|c|c|c|c|c|c|c|c|c|c|}
\hline Body & $I_{y}$ & $I_{z}$ & $P[\mathrm{~h}]$ & $P_{\phi}[\mathrm{h}]$ & $P_{\psi}[\mathrm{h}]$ & $P_{\mathrm{c}}[\mathrm{h}]$ & {$[\mathrm{K}-\mathrm{S}]_{\mathrm{RA}}$} & {$[\mathrm{K}-\mathrm{S}]_{\text {Dec }}$} & Comment \\
\hline Homogeneous & 1.83 & 1.98 & 12.40 & $\approx 9.7$ & $\approx 43.8$ & $\approx 17.3$ & - & - & $\begin{array}{l}\text { No relation with the obser- } \\
\text { vational RA/Dec periodicity } \\
\text { of } 270 \mathrm{~h}\end{array}$ \\
\hline
\end{tabular}

\begin{tabular}{|c|c|c|c|c|c|c|c|c|c|}
\hline $\begin{array}{l}\text { Slightly inho- } \\
\text { mogeneous } \\
\text { (near prolate). } \\
\text { Example. }\end{array}$ & 1.90 & 1.905 & 12.40 & $\approx 11.9$ & $\approx 270$ & $\approx 13$ & 0.5 & 0.02 & $\begin{array}{l}\text { No coincidence between } \\
\text { theoretical and observa- } \\
\text { tional periodograms of the } \\
\text { spin axis coordinates. }\end{array}$ \\
\hline $\begin{array}{l}\text { Inhomogeneous } \\
\text { case }\end{array}$ & $I_{y}$ & $0.96+0.99 I_{y}$ & 12.40 & $\approx 6.35$ & $\approx 13$ & $\approx 270$ & 0.94 & 0.82 & $\begin{array}{l}\text { Best set of parameters in } \\
\text { terms of the K-S probabil- } \\
\text { ity from RA data is different } \\
\text { from the best set of parame- } \\
\text { ters deduced from Dec data. } \\
\text { Inertia moments cannot be } \\
\text { constrained. }\end{array}$ \\
\hline
\end{tabular}

Mottola et al. (2014). In this study, simulations of slightly excited rigid-bodies fulfilling Euler equations under free-torque conditions were performed to interpret the periodicity detected in the coordinates of the spin axis. The main results of these simulations are summarized in Table 2.

From simulations considering slightly excited bodies, we found that Lomb periodograms of the RA/Dec distributions only show two significant periodicities. In ideal data, the most significant periodicity corresponds to the mean precession period, $P_{\phi}$. The second periodicity appearing in the periodograms always corresponds to $P_{\mathrm{c}}$ (Eq. (9)), a combination of the precession periodicity, $P_{\phi}$, and the nutation periodicity, the latter being half the intrinsic rotation period, $P_{\psi}$.

When ideal simulated data were perturbed by averaging over a period on the order of $P_{\phi}$ and noise was added, the significance was transferred from $P_{\phi}$ to $P_{\mathrm{c}}$, the latter becoming the most significant peak in the periodograms. The peak corresponding to $P_{\phi}$ may eventually disappear, leaving $P_{\mathrm{c}}$ as the single clearly detected periodicity.

When we assumed that the periodicity detected by Mottola is either the rotational period, $P=2 \pi / \Omega$, or the precessional period, $P_{\phi}$, then $P_{\psi}$, the spin around the principal axis corresponding to the highest inertia moment, might in principle be associated with the periodicity detected in observationally derived RA/Dec coordinates $(\approx 270 \mathrm{~h})$. This possibility was ruled out for two reasons. On the one hand, it would require that $67 \mathrm{P}$ must be nearly prolate from the standpoint of the inertia moments, which is unlikely because of the highly irregular shape of its nucleus. On the other hand, no single simulation showed the periodicity corresponding to $P_{\psi}$ in the periodograms. Lomb periodograms of simulated data in which the body is rotating with a $P_{\psi}$ around $270 \mathrm{~h}$ are quite different from the periodogram of the observationally derived data.

According to the results obtained from the simulations, the significant peak found in the periodograms of the observationally derived data could be interpreted as the combination $P_{\mathrm{c}}$ of periodicities $P_{\phi}$ and $P_{\psi}$. If the $12.40 \mathrm{~h}$ periodicity detected by Mottola et al. (2014) is considered the rotational period, $I_{y}$ and $I_{z}$ must follow the relation $I_{z}=0.96+0.99 I_{y}$. For this relation between inertia moments, the third inertia axis of the body (spin axis) would be precessing around the angular momentum vector with a mean period $P_{\phi} \approx 6.35 \mathrm{~h}$ and, at the same time, the body would be spinning around its third axis of inertia with a period $P_{\psi}=13.0 \mathrm{~h}$, being the rotational period, $P=2 \pi / \Omega$, around $12.4 \mathrm{~h}$.

Under these circumstances, a significant periodicity in the periodograms $P_{\mathrm{c}} \approx 270 \mathrm{~h}$ of the spin axis RA/Dec simulated coordinates was found, which became highly significant when noise and an averaging of $10 \mathrm{~h}$ (as in observations) were considered.

The ratio $I_{z} / I_{y}$ when the RA/Dec periodicity is identified with $P_{\mathrm{c}}$, regardless of the meaning of the periodicity detected by Mottola et al. (2014), is significantly higher than the ratio $I_{z} / I_{y}$ estimated assuming that $67 \mathrm{P}$ is homogeneous. If the RA/Dec periodicity around $270 \mathrm{~h}$ appearing in the periodograms is identified with $P_{\mathrm{c}}, 67 \mathrm{P}$ cannot have a homogenous density distribution.

To evaluate whether it is possible to constrain the inertia moments and excitation level, a systematic search of the probability of compatibility between simulated and actual RA/Dec patterns by means of two-sided K-S tests was performed. Even if it is possible to find very significant combinations of parameters $\left[I_{y}, I_{z}, E_{l}\right]$ when RA and Dec coordinates are considered separately, K-S probabilities when RA and Dec data are considered together do not allow constraining the inertia moments and excitation level.

Acknowledgements. OSIRIS was built by a consortium of the Max-Planck- Institut für Sonnensystemforschung, Göttingen, Germany, CISAS University of Padova, Italy, the Laboratoire d'Astrophysique de Marseille, France, the Instituto de Astrofísica de Andalucía, CSIC, Granada, Spain, the Research and Scientific Support Department of the European Space Agency, Noordwijk,The Netherlands, the Instituto Nacional de Técnica Aeroespacial, Madrid, Spain, the Universidad Politécnica de Madrid, Spain, the Department of Physics and Astronomy of Uppsala University, Sweden, and the Institut für Datentechnik und Kommunikationsnetze der Technischen Universität Braunschweig, Germany. The support of the national funding agencies of Germany (DLR), France(CNES), Italy(ASI), Spain(MEC), Sweden(SNSB), and the ESA Technical Directorate is gratefully acknowledged. P.J.G. and L.M.L. particularly acknowledge funding from projects AyA 2012-32237 and ESP 2014-54062-R. 


\section{References}

A'Hearn, M. F. 2011, ARA\&A, 49, 281

A'Hearn, M. F., Belton, M. J. S., Delamere, W. A., et al. 2011, Science, 332, 1396

Belton, M. J. S. 1990, Icarus, 86, 30

Bertaux, J.-L. 2015, A\&A, 583, A38

Bonev, B. P., Mumma, M. J., Radeva, Y. L., et al. 2008, ApJ, 680, L61

Burns, J. A., \& Safronov, V. S. 1973, MNRAS, 165, 403

Ciarletti, V., Levasseur-Regourd, A. C., Lasue, J., et al. 2015, A\&A, 583, A40

Dello Russo, N., Vervack, R. J., Weaver, H. A., et al. 2007, Nature, 448, 172

Feaga, L. M., A’Hearn, M. F., Sunshine, J. M., Groussin, O., \& Farnham, T. L. 2007, Icarus, 190, 345

Gibb, E. L., DiSanti, M. A., Magee-Sauer, K., et al. 2007, Icarus, 188, 224

Gross, R. S. 2000, Geophys. Res. Lett., 27, 2329

Jenkins, A. 2015, ArXiv e-prints [arXiv: 1506.02810]

Jorda, L., \& Gutiérrez, P. 2000, Earth Moon and Planets, 89, 135

Jorda, L., Gaskell, R., Capanna, C., Hviid, S., et al. 2016, Icarus, submitted

Kawakita, H., Furusho, R., Fujii, M., \& Watanabe, J.-I. 1997, PASJ, 49, L41

Keller, H. U., Barbieri, C., Lamy, P., et al. 2007, Space Sci. Rev., 128, 433

Keller, H. U., Mottola, S., Skorov, Y., \& Jorda, L. 2015, A\&A, 579, L5

Lomb, N. R. 1976, Ap\&SS, 39, 447

Luspay-Kuti, A., Hässig, M., Fuselier, S. A., et al. 2015, A\&A, 583, A4

Mottola, S., Lowry, S., Snodgrass, C., et al. 2014, A\&A, 569, L2

Pätzold, M., Andert, T., Hahn, M., et al. 2016, Nature, 530, 63

Press, W. H., Teukolsky, S. A., Vetterling, W. T., \& Flannery, B. P. 1992, Numerical recipes in $\mathrm{C}$. The art of scientific computing, 2nd edn. (Cambridge: University Press)

Preusker, F., Scholten, F., Matz, K.-D., et al. 2015, A\&A, 583, A33

Rickman, H., Marchi, S., A'Hearn, M. F., et al. 2015, A\&A, 583, A44

Samarasinha, N. H., \& Mueller, B. E. A. 2015, Icarus, 248, 347

Scholten, F., Preusker, F., Jorda, L., \& Hviid, S. 2015, http://www . sciops.esa.int/SB/PSA/docs/67P_C-G_reference-frames_and_ mapping-scheme_v2.pdf

Stacey, F. D., \& Davis, P. M. 2008, Physics of the Earth (Cambridge University Press)

Stellingwerf, R. F. 1978, ApJ, 224, 953

Vincent, J.-B., Bodewits, D., Besse, S., et al. 2015, Nature, 523, 63

1 Instituto de Astrofísica de Andalucía (CSIC), Glorieta de la Astronomía s/n. 18080 Granada, Spain

2 Aix-Marseille Université, CNRS, LAM (Laboratoire d'Astrophysique de Marseille) UMR 7326, 13388 Marseille, France

3 Planetary Science Institute, Tucson, Arizona 85719, USA

4 Department of Physics and Astronomy, Uppsala University, Box 516, 75120 Uppsala, Sweden

5 German Aerospace Center (DLR), Institut für Planetenforschung, Rutherfordstraße 2, 12489 Berlin, Germany

6 Institut für Geophysik und extraterrestrische Physik (IGEP), Technische Universität Braunschweig, Mendelssohnstraße 3, 38106 Braunschweig, Germany
7 IMCCE/Observatoire de Paris, 61 avenue de l'Observatoire, 75014 Paris, France

8 Centro de Astrobiología (INTA-CSIC), European Space Agency (ESA), European Space Astronomy Centre (ESAC), PO Box 78, 28691 Villanueva de la Cañada, Madrid, Spain

9 International Space Science Institute, Hallerstraße 6, 3012 Bern, Switzerland

10 Max-Planck-Institut für Sonnensystemforschung, Justus-vonLiebig-Weg 3, 37077 Göttingen, Germany

11 University of Padova, Department of Physics and Astronomy, Vicolo dell'Osservatorio 3, 35122 Padova, Italy

12 Scientific Support Office, European Space Research and Technology Centre/ESA, Keplerlaan 1, Postbus 299, 2201 AZ Noordwijk ZH, The Netherlands

13 PAS Space Research Center, Bartycka 18A, 00716 Warszawa, Poland

14 University of Maryland, Department of Astronomy, College Park, MD 20742-2421, USA

15 LESIA-Observatoire de Paris, CNRS, Université Pierre et Marie Curie, Université Paris Diderot, 5 place J. Janssen, 92195 Meudon Principal Cedex France

16 LATMOS, CNRS/UVSQ/IPSL, 11 boulevard d'Alembert, 78280 Guyancourt, France

17 Centro di Ateneo di Studi ed Attivitá Spaziali "Giuseppe Colombo" (CISAS), University of Padova, via Venezia 15, 35131 Padova, Italy

18 INAF, Osservatorio Astronomico di Padova, Vicolo dell'Osservatorio 5, 35122 Padova, Italy

19 CNR-IFN UOS Padova LUXOR, Via Trasea, 7, 35131 Padova, Italy

20 Department of Information Engineering, University of Padova, via Gradenigo, 6/B, 35131 Padova, Italy

21 University of Trento, via Sommarive, 9, 38123 Trento, Italy

22 Physikalisches Institut der Universität Bern, Sidlerstr. 5, 3012 Bern, Switzerland

23 INAF - Osservatorio Astronomico di Trieste, via Tiepolo 11, 34014 Trieste, Italy

24 Graduate Institute of Astronomy, National Central University, 300 Chung-Da Rd, 32054 Chung-Li, Taiwan

25 Operations Department, European Space Astronomy Centre/ESA, PO Box 78, 28691 Villanueva de la Cañada (Madrid), Spain

26 Solar System Exploration Research Virtual Institute, Southwest Research Institute, 1050 Walnut St., Suite 300, Boulder, CO 80302 , USA

27 Instituto Nacional de Tecnica Aeroespacial, Carretera de Ajalvir, p.k. 4, 28850 Torrejon de Ardoz, Madrid, Spain

28 Center for Space and Habitability, University of Bern, 3012 Bern, Switzerland

29 MTA CSFK Konkoly Observatory, 1121 Budapest, Hungary 